\title{
Chinese Herbal Medicines Attenuate Acute Pancreatitis: Pharmacological Activities and Mechanisms
}

\begin{abstract}
Hong Xiang ${ }^{1}$, Qingkai Zhang ${ }^{2}$, Bing $\mathrm{Qi}^{2}$, Xufeng $\mathrm{Tao}^{3}$, Shilin Xia ${ }^{4}$, Huiyi Song ${ }^{4}$, Jialin $Q u^{4}$ and Dong Shang ${ }^{1,2 *}$

'College (Institute) of Integrative Medicine, Dalian Medical University, Dalian, China, ${ }^{2}$ Department of General Surgery, The First Affiliated Hospital of Dalian Medical University, Dalian, China, ${ }^{3}$ College of Pharmacy, Dalian Medical University, Dalian, China, ${ }^{4}$ Clinical Laboratory of Integrative Medicine, The First Affiliated Hospital of Dalian Medical University, Dalian, China
\end{abstract}

Acute pancreatitis (AP) is a commonly occurring gastrointestinal disorder. An increase in the annual incidence of AP has been observed, and it causes acute hospitalization and high mortality. The diagnosis and treatment guidelines for AP recommend conservative medical treatments focused on reducing pancreatic secretion and secondary injury, as a primary therapeutic approach. Unfortunately, the existing treatment options have limited impact on the incidence and severity of AP due to the complex and multifaceted pathological process of this disease. In recent decades, Chinese herbal medicines (CHMs) have been used as efficient therapeutic agents to attenuate AP in Asian countries. Despite early cell culture, animal models, and clinical trials, CHMs are capable of interacting with numerous molecular targets participating in the pathogenesis of AP; however, comprehensive, up-to-date communication in this field is not yet available. This review focuses on the pharmacological activities of $\mathrm{CHMs}$ against $\mathrm{AP}$ in vitro and in vivo and the underlying mechanisms. A computational prediction of few selected and promising plant-derived molecules (emodin, baicalin, resveratrol, curcumin, ligustrazine, and honokiol) to target numerous proteins or networks involved in AP was initially established based on a network pharmacology simulation. Moreover, we also summarized some potential toxic natural products for pancreas in order to more safe and reasonable medication. These breakthrough findings may have important implications for innovative drug research and the future development of treatments for AP.

Keywords: acute pancreatitis, Chinese herbal formulas, natural products, pharmacological activities, toxic natural products

\section{INTRODUCTION}

Acute pancreatitis (AP) is characterized by a severe inflammatory response with the premature activation of pancreatic digestive enzymes, edema formation, cytoplasmic vacuolization, and infiltration of inflammatory cells into the pancreas (Yadav and Lowenfels, 2006; Banks et al., 2013). The most common risk for AP in adults is gallstones, which increases with age (Lowenfels et al., 2000). Excessive alcohol consumption, as the second-most common cause of AP after gallstones, has been shown to increase the risk of pancreatitis in a dose-dependent manner (Lankisch et al., 2002, 2015). The incidence of AP among subjects with long-term alcoholism is fourfold higher than 
those without (Yadav et al., 2007). Other causes include duct obstruction (e.g., related to a tumor or anatomic abnormalities), metabolic aberrations (e.g., hypertriglyceridemia), drug exposure (e.g., thiazides, azathioprine, and estrogens), smoking, and trauma (Yadav and Lowenfels, 2013; Lankisch et al., 2015) (Figure 1). Systemic diseases and trauma are particularly common in pediatric patients with AP and differ from those in adults (Snyder, 2000).

Acute pancreatitis is one of the most frequent gastrointestinal diseases leading to total hospital stays, with a reported global annual incidence of 13-45 per 100000 people (Lankisch et al., 2015). The number of discharges with AP as the principal diagnosis in the USA in 2009 increased 30\% compared with that in the year 2000 (Lankisch et al., 2015). Although nearly $80 \%$ of patients with AP exhibit mild symptoms and are easy to treat, $20 \%$ of those suffer a severe attack with progression to systemic inflammatory response syndrome (SIRS) and multiple organ dysfunction syndromes (MODS), and approximately 10-30\% of patients with severe acute pancreatitis (SAP) may die (Whitcomb, 2006). In addition, as shown in Figure 1, the associations between $\mathrm{AP}$ and certain chronic diseases have garnered attention in AP research in the past 10 years (Yadav et al., 2012; Gillies et al., 2016; Kennedy et al., 2016). Investigations from the US showed a transition to chronic and pancreatic cancer from AP in 32.3\% after 3.4 years (Yadav et al., 2012). Findings from a crosssectional follow-up study and a recent comprehensive systematic review indicate that approximately $40 \%$ of patients may develop newly diagnosed prediabetes (e.g., chronic hyperglycemia, insulin resistance) or diabetes after AP, with the risk of diabetes doubling within 5 years (Gillies et al., 2016; Kennedy et al., 2016). Therefore, approaches with high efficacy and minimal side effects are imperative for the treatment of AP.

Since the first international classification of pancreatitis formulated during the 1963 Marseille meeting, the management guidelines of AP have been enacted one after another; so far, a preliminary consensus regarding the treatment for AP has been established (Tenner et al., 2013; Working Group IAP/APA Acute Pancreatitis Guidelines, 2013; Yokoe et al., 2015). Except for a few acute hemorrhagic necrotizing pancreatitis (AHNP) cases that require surgery, the primary therapeutic approach is to recommend conservative treatment focused on reducing pancreatic secretion and secondary injury, including fasting, fluid resuscitation, protease inhibitors, and antibiotics (Whitcomb, 2006; Banks et al., 2013; Lankisch et al., 2015). Although these strategies have been verified in randomized controlled trials, they have a limited impact on the incidence and severity of AP due to their unpredictable side effects and poor patient compliance. So far, AP is still a significant and unresolved challenge to clinicians. Hence, there is a huge demand to explore novel candidates for AP treatment.

Chinese herbal medicines (CHMs), which are abundant sources of biologically active substances, have been commonly used in clinical practices in many countries (Normile, 2003). Presently, more and more CHMs (including Chinese herbal formulas and pleiotropic natural products) have been discovered to have potent effects against AP through targeting numerous protein or biological networks involved in this disease (Tian et al.,
2009; Chen et al., 2011; Xiang et al., 2016). Thus, CHMs are promising candidate drugs for the treatment of AP compared with western medicine, which usually focuses on a single target. For the future development of innovative medicines and to extend the influence of CHMs worldwide, we systematically summarize the available Chinese herbal formulas and pleiotropic natural products used in the treatment of AP and discuss their underlying mechanisms in this review. Within this frame, the underlying mechanisms of AP will also be covered.

\section{UNDERLYING MECHANISMS OF AP}

For centuries, many theories have been proposed to explain the underlying mechanism of AP. As presented in Figure 2, the pathogenesis of AP seems to be related to a series of complex and multifaceted pathological processes, involving pancreatic selfdigestion (Lerch and Gorelick, 2000), inflammatory response, oxidative stress (Xiang et al., 2016), intracellular calcium overload, endoplasmic reticulum (ER) stress (Wu J.S. et al., 2016), pancreatic acinar cell apoptosis and necrosis (Xiang et al., 2016), and microcirculation disorder (Tomkotter et al., 2016). Current therapies, which target the above pathological mechanisms, may improve the prognosis of AP.

\section{Pancreatic Self-digestion Theory}

The "pancreatic self-digestion theory" was proposed by Chiara et al. (1886) for the first time (Jin et al., 2013), and this theory suggested that pancreatic duct obstruction and the blockage of pancreatic juice outflow under pathological conditions are associated with exocrine pancreatic hyperstimulation and active trypsin reflux and result in the autodigestion of the pancreas and pancreatitis (Lerch and Gorelick, 2000). This theory was supported by research executed in cerulean-induced AP mice with or without the trypsinogen isoform 7 (T7) gene $\left(\mathrm{T}^{-} /^{-}\right)$. Unlike zymogen activation and AP in wild-type mice, necrosis and cell death were significantly reduced in $\mathrm{T}^{-} /^{-}$mice (Dawra et al., 2011). Most researchers accept that the direct trigger for the onset and exacerbation of AP relates to the inappropriate activation of trypsinogen to trypsin, the key enzyme in the activation of additional zymogens, and a lack of prompt elimination of active zymogens (Lerch and Gorelick, 2000; Whitcomb, 2006). Pancreatic acinar cells are specialized for the production, storage, and release of pancreatic zymogens (Hofbauer et al., 1998). During AP, lysosomal enzymes are mistargeted to the organelles containing zymogens within the acinar cell. The lysosomal hydrolase cathepsin-B prematurely activates trypsinogen, whereas the inhibition or knockout of cathepsin-B has been shown to relieve trypsinogen activation and acinar cell damage (Saluja et al., 1997; Van Acker et al., 2002).

In addition, there are a variety of protective mechanisms against premature zymogen activation in vivo through the inhibition or degradation of activated trypsin (Hirota et al., 2006; Binker et al., 2015). These mechanisms include inhibition by pancreatic secretory trypsin inhibitor (PSTI), also known as a serine protease inhibitor Kazal-type 1 (SPINK1) or 


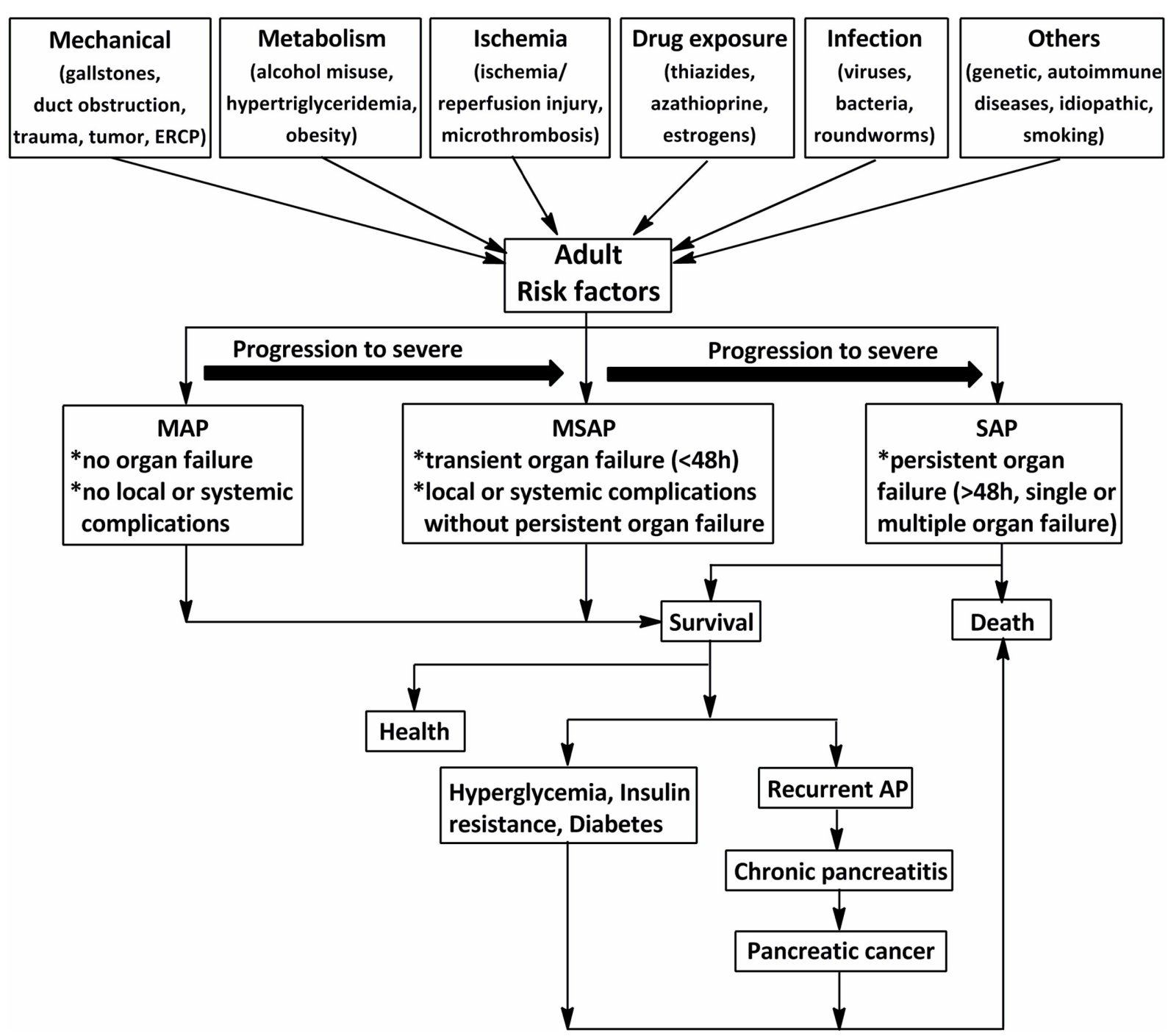

FIGURE 1 | The schematic of the procedure of acute pancreatitis (AP) in adults (>18 years). The common risks for AP in adults are mechanical, metabolism, ischemia, drug exposure, infection, and so on. According to the 2012 revision of the Atlanta classification and definitions, AP should be divided into mild acute pancreatitis (MAP), moderately severe acute pancreatitis (MSAP), and severe acute pancreatitis (SAP). Although MAP and MSAP account for nearly $80 \%$ of AP cases, $20 \%$ of patients with SAP suffer persistent organ failure or even death. Patients with AP often develop prediabetes, diabetes and/or other complications after discharge from hospital. ERCP, endoscopic retrograde cholangiopancreatography.

tumor-associated trypsin inhibitor (TATI) (Hirota et al., 2006), and degradation by chymotrypsin-C (CTRC) (Binker et al., 2015) and the lysosomal hydrolase cathepsin-L (Wartmann et al., 2010). Once these protective mechanisms are expended, there is an increased risk of developing AP.

\section{Inflammatory Response}

An acute inflammatory cascade is shown to be the main reason that mild acute pancreatitis progresses to SIRS and MODS in response to pancreatic cell injury (Whitcomb, 2006; Lankisch et al., 2015). In recent years, studies suggested that damage associated molecular patterns (DAMPs), mainly high mobility group box 1 (HMGB1) protein, are released by injured and necrotic acinar cells (Scaffidi et al., 2002). HMGB1 is an intracellular DNA-binding protein involved in neutrophil activation and pro-inflammatory factor secretion via Toll-like receptors (TLRs) in the pathogenesis of AP (Shen and Li, 2015). TLR4 is the first target response to extracellular HMGB1 and can activate the myeloid differentiation primary response gene 88 (Myd88)-dependent pathway, TNF-associated factor 6 and the MAPK signal transduction pathway, which then lead to the activation of nuclear factor kappa-B (NF- $\kappa \mathrm{B})$ and activator protein-1 (AP-1) (Li et al., 2016b). NF- $\kappa$ B and AP-1 are known transcription factors with multiple functions, required for the early regulation of inflammatory signaling (Yang et al., 2016). $\mathrm{NF}-\kappa \mathrm{B}$ in the cell cytoplasm is bound to the inhibitor protein kappa B (ІкB) in an inactive form. During cellular stress, IкB is phosphorylated by specific IКB kinase (IKK) and rapidly 


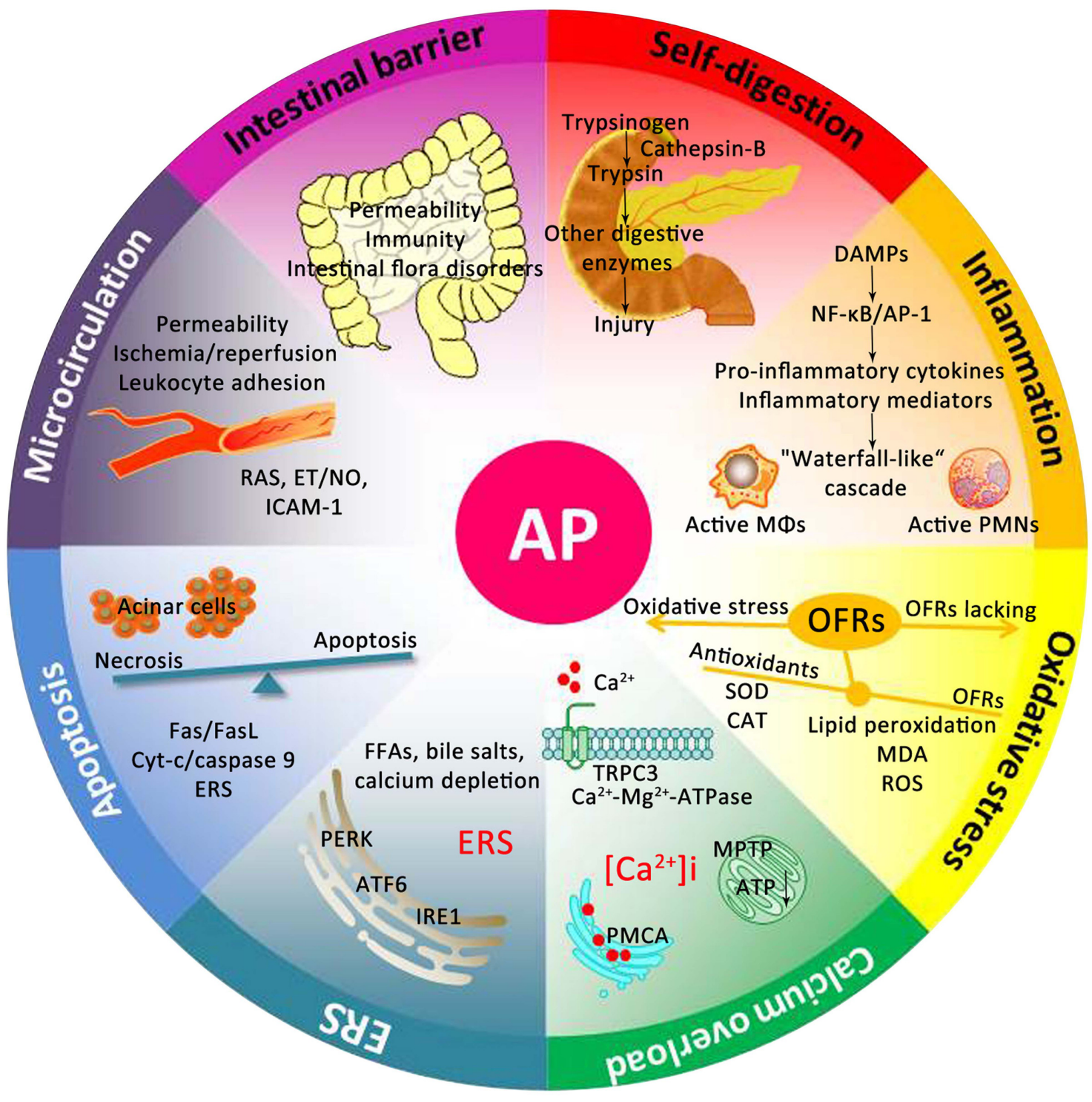

FIGURE 2 | Underlying mechanisms of the occurrence and development of AP. DAMPs, damage associated molecular patterns; NF-кB, nuclear factor kappa-B; AP-1, activator protein-1; MФs, macrophages; PMNs, polymorphonuclear neutrophils; OFRs, oxygen free radicals; SOD, superoxide dismutase; CAT, catalase; MDA, malondialdehyde; ROS, reactive oxygen species; TRPC3, transient receptor potential channel 3; MPTP, mitochondrial permeability transition pore; ATP, adenosine triphosphate; PMCA, plasma membrane calcium ATPase; FFAs, free fatty acids; ERS, endoplasmic reticulum stress; PERK, RNA-activated protein kinase-like ER kinase; ATF6, activation transcription factor 6; IRE1, inositol requiring protein 1; Cyt-c, Cytochrome c; RAS, renin-angiotensin system; ET, endothelin; $\mathrm{NO}$, nitric oxide; ICAM-1, intercellular adhesion molecule-1.

degraded via the proteasome-dependent pathways (Hayden and Ghosh, 2008). NF-kB activation during AP is capable of upregulating the expression of cytokines, chemokines and adhesion molecules, the activation of macrophages, and the infiltration of neutrophils and lymphocytes into the pancreas and peritoneum, thus amplifying the inflammatory response (Jakkampudi et al., 2016). AP-1 is a heterodimer complex formed from proteins belonging to the c-Fos, c-Jun, and activating transcription factor (ATF) families (Schraml et al., 2009). AP-1 can be activated by multiple factors such as growth factors, cytokines, and chemokines, which often act in concert with NF- $\mathrm{B}$ to control the cascade chain reaction of inflammatory mediators (Koh et al., 2010). The mechanism of AP-1 activation involves the phosphorylation of c-Jun (Schraml et al., 2009).

During AP, neutrophils migrate from the bloodstream to the damaged tissues, significantly increasing the numbers of local neutrophils in the pancreas (Yang Z.W. et al., 2015). Activated neutrophils have the capability to exacerbate the release of many types of pro-inflammatory cytokines and inflammatory mediators, which contain tumor necrosis factor- $\alpha$ (TNF- $\alpha$ ), interleukin (IL)-1, IL-6, and IL-8, oxygen free radicals (OFRs), platelet-activating factor (PAF), leukotrienes, and thromboxane 
A2 (TXA2), and many enzymes, in particular, elastase and phospholipase A2 (PLA2) (Kolaczkowska and Kubes, 2013; Nauseef and Borregaard, 2014; Yang Z.W. et al., 2015). These released signaling molecules, in turn, activate NF-kB via highly specific receptor binding patterns, leading to pancreatic inflammation, necrosis and microcirculation disorder, and the release of excess endotoxin (Rotstein, 2014). The inflammatory response is constantly activated, not only in the local pancreas but also in the extrapancreas, and evolves into multiple organ injury and SIRS.

\section{Oxidative Stress}

Kishimoto et al. (1995) found that pancreatic OFRs increased after the induction of AP in rats, demonstrating that significant peroxidation occurs in AP. In patients with AP, the involvement of OFRs is independent of the underlying etiology (Park et al., 2003). Upon physiological conditions, the production and elimination of OFRs remain balanced. OFRs accumulate in the pancreas during AP development, which can attack macromolecules, such as proteins, lipids, and polysaccharides inside the biomembrane, initiating lipid peroxidation and resulting in the breakage of membrane stability and the release of zymogen granules in the acinar cell (Chvanov et al., 2005; Perez et al., 2015). OFRs activate PLA that can break down lecithinum inside the cellular membrane and further initiate pancreatic edema, hemorrhage, degeneration, and necrosis (Tsukahara et al., 1999). Moreover, many OFRs released in AP also cause NF- $\kappa B$ and AP-1 activation, antioxidant consumption, and superoxide dismutase (SOD) activity decrease, which in turn causes lipid peroxidation and pancreas damage (Yu and Kim, 2014; Perez et al., 2015).

\section{Calcium Overload}

In general, calcium release, uptake and extrusion mechanisms remain in fine coordination, whereas some factors can influence calcium regulation and increase the intracellular calcium concentration under certain conditions (Criddle, 2016). Cytosolic calcium is a secondary messenger of intracellular signal transduction and applies to many cellular events, including the regulation of trypsin activity (Kruger et al., 2000). In AP, calcium homeostasis is disrupted, resulting in calcium overload within a cell, which can aggravate the disease state (Kruger et al., 2000; Maleth and Hegyi, 2014). Studies have shown that hypercalcemia causes histological damage during AP in a time- and dose-dependent manner (Gerasimenko et al., 2014). Calcium overload within the pancreatic acinar cells can induce mitochondrial impairment through the formation of the mitochondrial permeability transition pore (MPTP) and the depletion of ATP and necrosis, thus leading to serious injury of the acinar cells and pancreatic inflammation (Mukherjee et al., 2016).

The maintenance of intracellular calcium homeostasis depends on a stable mechanism that coordinates calcium release, entry and exit (Criddle, 2016). It is known that calcium release from internal stores occurs via inositol 1,4,5-trisphosphate receptor (IP3R)-dependent calcium oscillations that promote exocrine secretion (Camello-Almaraz et al., 2006). There are several ion channels (TRPC3 channels, STIM1-Orai complex) in pancreatic acinar cells implicated in important calcium entry mechanisms, which mediate sustained pathophysiological elevations in intracellular calcium causing mitochondrial injury and cell death (Kim et al., 2009; Lur et al., 2009). Considering the benefit of the production and supply of intracellular adenosine triphosphate (ATP) for intracellular calcium homeostasis in the pancreatic acinar cell, recent studies reported that calcium clearance requires ATP-dependent pumps modulated by sustained oxidative stress (Bruce and Elliott, 2007). In AP, the structural integrity of the plasma membrane is destroyed, and the quantity and activity of endocytoplasmic/sarcoplasmic reticulum (SR) membrane $\mathrm{Ca}^{2+}$-ATPase (PMCA) and cytomembrane $\mathrm{Ca}^{2+}-\mathrm{Mg}^{2+}$-ATPase are decreased, and the intracellular calcium is not pumped out of the cell or back into the calcium stores in time (Baggaley et al., 2008; Wang et al., 2008).

\section{ER Stress}

The acinar cells of the exocrine pancreas have an abundant ER that contributes to the synthesis and secretion of proteins. ER enzymes require optimal ion concentrations (e.g., calcium) and redox conditions for their regulation and function (Kubisch and Logsdon, 2008). Excess free fatty acids (FFAs), bile salts, calcium depletion of the ER stores, and oxidative stress activate the ER stress response and the injury of acinar cells, which initiates a complicated cascade of events during the early stage of AP (Zeng et al., 2012; Wu et al., 2013). RNA-activated protein kinase-like ER kinase (PERK), activation transcription factor 6 (ATF6), and inositol requiring protein 1 (IRE1) are believed to be ER stress sensors, which play a key role in transducing the stress signals from the ER to the cytoplasm (Kubisch and Logsdon, 2008). In $\mathrm{AP}$, these signaling molecules and their downstream molecules TNF receptor-associated factor 2 (TRAF2) and apoptosis signalregulating kinase 1 (ASK1) are highly activated (Sendler et al., 2013). This activation parallels trypsinogen activation and leads to apoptosis and inflammatory and immune responses (Kubisch and Logsdon, 2007).

\section{Cell Apoptosis}

Both necrosis and apoptosis are the two types of crucial cell death in AP, and may interchange under appropriate conditions. Recent studies prove that the regulation of the necrosis/apoptosis switch is beneficial for alleviating the severity of AP because apoptotic cells maintain membrane integrity without triggering an inflammatory cascade, unlike necrotic cells, which release intracellular contents containing pro-inflammatory and immunogenic cytokines (Takeyama, 2005; Fu et al., 2016). Apoptosis is controlled via three known pathways: the death receptor, the mitochondrial pathway, and the ER stress-induced apoptotic pathway. In early SAP, the stimulation of death receptors such as Fas and tumor necrosis factor receptor 1 (TNFR1) leads to the formation of a death-inducing signaling complex and initiator caspase-8, which initiates downstream caspase-3 (Zhang et al., 2007a). Death receptors transmit the death signals to mitochondria resulting in the release of cytochrome $c$ (a precursor of apoptosis) into the cytoplasm through a permeability transition pore in the mitochondrial 
membrane, which subsequently binds to the apoptotic protease activating factor-1 (Apaf-1) and procaspase-9 to form the apoptosome and activate caspase-3 (Xia et al., 2002). Bcl-2, an anti-apoptotic member of the Bcl-2 family, can break the opening of the permeability transition pore induced by Bax (Ma et al., 2011). Recent studies suggest several molecules such as IRE1, $\mathrm{C} / \mathrm{EBP}$ homologous protein (CHOP), Bcl-2 family and caspase12 play a role in ER stress-induced apoptosis (Morishima et al., 2002). Evidence indicates that the sustained activation of IRE1 enhances cell survival, suggesting a link between ER stress and death cell fate. CHOP transcription is induced by the activation of PERK and ATF6. Apoptosis via the CHOP signaling pathways is mitochondria-dependent because CHOP can break down the membrane potential and promote the release of cytochrome $c$ from mitochondria (Kubisch and Logsdon, 2008). The members of the ER-resident proapoptotic Bcl-2 family, Bak and Bax, can maintain the homeostasis of ER calcium. When ER stress and increased cytosolic calcium correlate with an increase in calpain activation, it contributes to the activation of ER-resident caspase12 , which directly excites caspase- 3 and leads to cellular apoptosis (Nakagawa and Yuan, 2000). Bcl-2 can clear OFRs and prevent the release of calcium from ER and leads to necrosis by inhibiting apoptosis (Wu and Tang, 2016).

\section{Microcirculation Disorder}

Microcirculation obstruction throughout the development of AP is a systemic response to pancreatic injury and is closely associated with MODS (Zhou and Chen, 2002; Ma et al., 2011; Tomkotter et al., 2016). The pancreas is the main site of the abnormal metabolism of eicosanoids (TXA2 and PGI2), which are decomposed into several types of inflammatory mediators, such as PAF and leukotrienes, thereby aggravating the inflammatory reaction and microcirculatory disturbance (Zhang et al., 2009b). Microcirculatory hypoperfusion causes the inflow of calcium into cells, leading to calcium overload in the pancreatic cells (Zhou et al., 2004). Calcium influx activates the phospholipid cell system, leading to the destruction of the lysosomal membrane and the release of the enzyme and large amounts of toxic media (Maleth and Hegyi, 2014). In addition, significant hemorrhagic changes in a rat model of SAP have been reported, including high hematocrit (HCT) and blood viscosity, as well as microthrombus formation. The possible mechanism relates to the activation of pancreatin that increases the permeability of capillary vessels, resulting in the exudation of lots of plasma-like liquids from the blood circulation to tissue space. Fragmented erythrocytes release tissue factors, such as adenosine diphosphate (ADP), which can activate the coagulation system and consume abundant fibrinogen (Ma et al., 2011). These factors may be the primary causes of progressive pancreatic tissue necrosis and MODS.

\section{Altered Gut Barrier Permeability}

The gut mucosal barrier serves as a "natural guard" in the human body, preventing the entrance of potentially harmful intestinal bacteria and endotoxin into the systemic circulation and extra-intestinal tissues. The gut acts as an important target organ in response to SAP, and as the main source of pancreatic bacterial super infection and related septic complications (Wang et al., 2016). Gram-negative enteric-type organisms are shown to be the culprit in most pancreatic and peripancreatic infections (Hanna et al., 2014). In SAP early, hypovolemia, splanchnic vasoconstriction, and ischemia-reperfusion injury initiates a derangement in gut barrier function. Increased intestinal permeability, physical and chemical factor changes, and cell immune dysfunction induced by impaired gut mucosa barrier destroy normal flora structure and promote intestinal lumenderived bacteria and endotoxins translocation to other organs through blood circulation or the lymphatic system, or through a pancreatic duct or bile duct directly connected with the intestinal tract (Guo et al., 2014). Intestinal microflora can also directly penetrate the damaged intestinal mucosal barrier into the abdominal cavity. In this scenario, an augmented immune response is triggered, which causes the gut to become a proinflammatory organ that releases cytokines, chemokines, and other pro-inflammatory intermediates. These mediators cause a "waterfall-like" inflammation cascade via the excessive activation of macrophages and neutrophils and release of oxidant and proteolytic enzymes, which aggravates secondary infection in the pancreas, eventually resulting in SIRS and MODS (Leveau et al., 1996; Wu et al., 1998).

\section{CHINESE HERBAL FORMULAS}

Chinese herbal formulas have upheld the holistic therapeutic philosophy for thousands of years, which consists of two or more appropriate medicinal plants or animals according to the prescription compatibility principle of traditional Chinese medicine (TCM) formulations and determining the dosage and usage of each medicine (Normile, 2003; Yao et al., 2016b). The components of Chinese herbal formulas are complex and diverse, and the main treatment mechanism seems reasonable. These herbal formulas are an organic combination of many effective components having a multi-target effect on the disease in the body by multiple pathways (Cheng et al., 2015; Wu T.Y. et al., 2016; Xiang et al., 2016). An increasing number of Chinese herbal formulas have been reported to have significant anti-AP effects, and have become a treatment option in many hospitals for AP (Table 1).

Dachengqi decoction (DCQD), first documented in "Shang Han Lun" (Treatise on Febrile Diseases), is a representative purgative for constipation treatment and for clearing internal heat in the gastrointestinal tract. DCQD consists of Radix et Rhizoma Rhei, Cortex Magnoliae Officinalis, Fructus Aurantii Immaturus, and Natrii Sulphas, which have been used as a classical prescription in China to treat AP for more than three decades (Chen et al., 2010). Rhein, naringin, and honokiol may be the major effect components of DCQD in treatment of AP (Zhang et al., 2016). Clinical studies indicated that DCQD could decrease the ratio of lactulose $(\mathrm{L}) / \mathrm{mannitol}(\mathrm{M})$ and the surrogate of intestinal permeability assays, which suggested that DCQD protect the intestinal mucosal immune barrier and decrease the incidence of pancreatic infection and MODS (Chen et al., 2010; Jiang, 2010). 
TABLE 1 | List of traditional Chinese medicine formulas for the treatment of AP.

\begin{tabular}{|c|c|c|c|}
\hline Formula & Common composition & Mechanisms & Reference \\
\hline $\begin{array}{l}\text { DCQD } \\
\text { (Modified) }\end{array}$ & $\begin{array}{l}\text { Radix Rhei Et Rhizome (Dahuang), Thenardite (Xuanmingfen), } \\
\text { Magnolia Officinalis Rehd Et Wils (Houpu), Aurantii Fructus } \\
\text { (Zhiqiao), Persicae Semen (Taoren), Raphani Semen (Laifuzi) }\end{array}$ & $\downarrow H M G B 1, T N F-\alpha$ & Qin et al., 2015 \\
\hline CQCQD & $\begin{array}{l}\text { Radix Bupleuri (Chaihu), Scutellariae Radix (Huangqin), } \\
\text { Magnolia Officinalis Rehd Et Wils (Houpu), Aurantii Fructus } \\
\text { Immaturus (Zhishi), Artemisiae Scopariae Herba (Yinchen), } \\
\text { Fructus Gardeniae (Zhizi), Radix Rhei Et Rhizome (Dahuang), } \\
\text { Natrii Sulfas (Mangxiao) }\end{array}$ & $\begin{array}{l}\uparrow S E R C A 2 \\
\downarrow N F-\kappa B, \text { TNF- } \alpha, \text { IL6 } \\
\downarrow\left[\mathrm{Ca}^{2+}\right] i \\
\downarrow C C K R 1, \text { PLC, IP3 } \\
\uparrow C y t o c h r o m e ~ c, \text { Caspase-3 } \\
\uparrow n A C h R a l p h a 7, \text { Ach, } \downarrow \text { IL6 }\end{array}$ & $\begin{array}{l}\text { Xue et al., } 2008 \\
\text { Li et al., } 2008 \\
\text { Deng et al., } 2008 \text { a } \\
\text { Guo et al., } 2015 \\
\text { Lin et al., } 2014 \\
\text { Xue et al., } 2014\end{array}$ \\
\hline QYD & $\begin{array}{l}\text { Gardenia jasminoides Ellis (Zhizi), Cortex Moutan (Danpi), Radix } \\
\text { Paeoniae Rubra (Chishao), Aucklandiae Radix (Muxiang), } \\
\text { Magnolia Officinalis Rehd Et Wils (Houpu), Corydalis Rhizoma } \\
\text { (Yuanhu), Radix Rhei Et Rhizome (Dahuang), Natrii Sulphas } \\
\text { (Mangxiao) }\end{array}$ & $\begin{array}{l}\downarrow \text { Genes: Rgs2, Pnlip, Cpa2, Ela2, } \\
\text { LOC503278, Sv2b, LOC500909, Cln3, } \\
\text { Reg1, Fbxl20 } \\
\uparrow \text { Genes: Glrx1, LOC499457, Txn12, } \\
\text { Eef1g, LOC499793, Rpl10, } \\
\text { LOC499906, Dap, Eef1b2, LOC362290 }\end{array}$ & Zhu et al., 2014 \\
\hline QYD (Modified) & $\begin{array}{l}\text { Radix Rhei Et Rhizome (Dahuang), Natrii Sulphas (Mangxiao), } \\
\text { Kansui Radix (Gansui), Radix Bupleuri (Chaihu), Aucklandiae } \\
\text { Radix (Muxiang), Scutellariae Radix (Huangqin), Coptidis } \\
\text { Rhizoma (Huanglian), Magnolia Officinalis Rehd Et Wils (Houpu), } \\
\text { Gardenia jasminoides Ellis (Zhizi), Paeoniae Radix Alba } \\
\text { (Baishao), Persicae Semen (Taoren) }\end{array}$ & $\downarrow N F-\kappa B, T N F-\alpha, I L-6, \mid L-8$ & Yang et al., 2009 \\
\hline YCHD & $\begin{array}{l}\text { Artemisia capillaris Thunb. (Yinchen), Gardenia jasminoides Ellis } \\
\text { (Zhizi), Rheum officinale Baill. (Dahuang) }\end{array}$ & $\uparrow P P A R \gamma, \downarrow N F-\kappa B$ & Xiang et al., 2016 \\
\hline LHD & $\begin{array}{l}\text { Radix Rhei Et Rhizome (Dahuang), Cortex Phellodendri } \\
\text { Chinensis (Huangbai), Rhizoma Bletillae (Baiji), Radix Angelicae } \\
\text { Dahuricae (Baizhi), Fructus Mume (Wumei), Herba Menthae } \\
\text { (Bohe) }\end{array}$ & $\downarrow T N F-\alpha$, IL-6, IL-10, $\uparrow S O D$ & Peng et al., 2013 \\
\hline TXHYD & $\begin{array}{l}\text { Radix Rhei Et Rhizome (Dahuang), Natrii Sulfas (Mangxiao), } \\
\text { Aurantii Fructus Immaturus (Zhishi), Curcumae Radix (Yujin), } \\
\text { Polygoni Cuspidati Rhizoma Et Radix (Huzhang), Cortex } \\
\text { Moutan (Mudanpi), Radix Paeoniae Rubra (Chishao), Corydalis } \\
\text { Rhizoma (Yanhusuo) }\end{array}$ & $\downarrow E T$ & Fang et al., 2007 \\
\hline \multirow[t]{2}{*}{ YHQYD } & $\begin{array}{l}\text { Radix Rhei Et Rhizome (Dahuang), Natrii Sulfas (Mangxiao), } \\
\text { Scutellariae Radix (Huangain), Paeoniae Radix Alba (Baishao), } \\
\text { Radix Ophiopogonis (Maidong), Aucklandiae Radix (Muxiang), }\end{array}$ & $\uparrow$ Pancreatic blood flow & Chen et al., 2011 \\
\hline & $\begin{array}{l}\text { Radix Bupleuri (Chaihu), Semen Arecae (Binglang), Cortex } \\
\text { Meliae (Kulianpi), Fructus Quisqualis (Shijunzi), Rhizoma } \\
\text { Chuanxiong (Chuanxiong), Salviae Miltiorrhizae (Danshen), } \\
\text { Radix Astragali (Huangqi), Radix Ginseng (Renshen) }\end{array}$ & & \\
\hline
\end{tabular}


TABLE 1 | Continued

\begin{tabular}{|c|c|c|c|}
\hline Formula & Common composition & Mechanisms & Reference \\
\hline $\mathrm{CHSHD}$ & $\begin{array}{l}\text { Radix Bupleuri (Chaihu), Radix Rhei Et Rhizome (Dahuang), } \\
\text { Scutellariae Radix (Huangqin), Coptidis Rhizoma (Huanglian), } \\
\text { Phellodendri Chinensis Cortex (Huangbai), Radix Paeoniae } \\
\text { Rubra (Chishao), Radix Salviae (Danshen) }\end{array}$ & $\downarrow T N F-\alpha, I L-6, \uparrow \mid L 10$ & Wang H. et al., 2009 \\
\hline
\end{tabular}

DCQD, Dachengqi decoction; CQCQD, Chaiqinchengqi decoction; QYD, Qingyi decoction; QYG, Qingyi granule; YCHD, Yinchenhao decoction; LHD, Liuhedan; TXHYD, Tongxiahuayu decoction; YHQYD, Yihuoqingyi decoction; CHSHD, Chaihusihuang decoction; CRP, C-reactive protein; IL, interleukin; TNF- $\alpha$, tumor necrosis factor- $\alpha$; L/M, lactulose/mannitol; LPS, lipopolysaccharide; ROS, reactive oxygen species; NO, nitric oxide; iNOS, inducible nitric oxide synthase; HMGB1, high mobility group box 1; TLRs, Toll-like receptors; NF- $\kappa$ B, nuclear factor kappa-B; p38 MAPK, p38 mitogen-activated protein kinases; SAA, serum amyloid A; MMP-9, matrix metalloproteinase 9; SERCA2, sarco/endoplasmic reticulum Ca ${ }^{2+}$-ATPase; CCKR1, cholecystokinin receptor 1; PLC, phospholipase C; IP3, inositol-1,4,5-triphosphate; nAChRalpha7, neuronal acetylcholine receptor alpha 7; Ach, acetylcholine; PPAR , peroxisome proliferator-activated receptor gamma; SOD, superoxide dismutase; ET, endothelin.

On the molecular mechanism, DCQD decreases proinflammatory cytokines and alleviates the severity of AP through inhibiting TLR/HMGB-1 signal pathways (Chen et al., 2010, 2015; Qin et al., 2015). DCQD could also render pancreas more resilient to stress and microcirculation disorder through eliminating excessive reactive oxygen species (ROS), inducing apoptosis and relieving the necrosis in acinar cells (Ren et al., 2009; Wang et al., 2012). Moreover, recent studies have shown that DCQD is an effective digestive kinetic agent that could promote the gastrointestinal motility and the recovery of intestinal mucosal permeability, and affect the bacterial translocation in the animal models of AP (Chen et al., 2010; Qin et al., 2015).

Chaiqinchengqi decoction (CQCQD), modified from DCQD, is a traditional Chinese prescription used as a purgative. In recent decades, CQCQD has shown significant efficacy in the treatment of AP both in vivo and in vitro (Liu et al., 2004; Deng et al., 2008a; Xue et al., 2008; Wang et al., 2011). Clinical trials have also proven that CQCQD significantly relieves the severity of clinical symptoms, reduces the duration of organ damage, and shortens the hospitalization time of AP patients (Wang et al., 2011). Research showed that CQCQD may have efficient actions on the activation of choline acetyl transferase (ChAT) and neuronal acetylcholine receptor alpha 7 (nAChR $\alpha 7)$ in peritoneal macrophages, which could inhibit the release of active macrophage pro-inflammatory cytokines (Li et al., 2008; Wang et al., 2011; Xue et al., 2014; Wu W.U. et al., 2016). CQCQD also inhibit the exocrine function of the pancreatic acinar cells and relieve pancreatic tissue lesions via reducing the overload of intracellular calcium (Deng et al., 2008a,b; Xue et al., 2008; Guo et al., 2015). In addition, CQCQD also regulates necrosis to apoptosis in pancreatic cells by promoting the release of mitochondrial cytochrome $c$ and increasing pancreatic caspase3 activity in SAP rats (Lin et al., 2014). Moreover, CQCQD has a protective effect in SAP complicated with acute lung injury (ALI) and acute respiratory distress syndrome by inhibiting ER stress in AMs, attenuating pro-inflammatory cytokine release and paracellular leakage, which involved in the down-regulation of p-Src, p-p85 $\alpha$, and c-Fos (Wang Z.C. et al., 2009; Jia et al., 2015; Wu W.U. et al., 2016). In addition, the modified CQCQD can relieve the severity of clinical symptoms in patients with SAP via lowering serum amyloid A (SAA) and matrix metalloproteinase 9 (MMP-9) (Wu et al., 2012; Guo et al., 2013).
Another common Chinese herbal decoction, qingyi decoction (QYD) or qingyi granule (QYG) is generally well tolerated by patients and exhibits purgative function, eliminates blood stasis, promotes blood circulation, and reduces inflammation in the pathogenesis of AP (Yang et al., 2009, 2013; Zhu et al., 2014). Moreover, clinical research proved that QYD could ameliorate AP-induced intestinal barrier injury by inhibiting the expression of intestinal secreted phospholipase A2 (sPLA2) (Zhang et al., 2015). Furthermore, an Illumina whole genome expression profile analysis for pancreatic RNA expression of SAP rats screens 575 differential genes between the SAP and QYD group, including 92 up-regulated genes and 483 down-regulated genes; and the Gene Ontology (GO) categories indicated that these genes are involved the MAPK and NLR signaling pathways, metabolic pathways, cell cycle, and oxide reductase activities (Yang et al., 2013; Zhu et al., 2014). In addition, QYD can reduce the extent of extrapancreatic organ injuries in SAP. QYD administration not only reduces SAP-induced ALI by reducing alveolar type II epithelial cell (AEC II) apoptosis, inhibiting the overexpression of secretory type II phospholipase A2 (sPLA2) (Liu et al., 2014), but also improves SAP complicated liver and renal injuries through decreasing HMGB1 expression (Yang Y.S. et al., 2015). In addition, the actions of modified QYD involve in the biological processes include directly neutralizing endotoxins, reducing intestinal endotoxin generation and absorption, inhibiting excessive neutrophil activation and NF$\kappa \mathrm{B}$ expression, and minimizing the release of inflammatory cytokines (Yang et al., 2009).

Moreover, our previous study found that Yinchenhao decoction (YCHD), known as an anti-inflammatory and choleretic agent, may also be a potential therapy for AP through pro-apoptosis, anti-inflammation, anti-oxidation, and regulation of lipid metabolism partially via regulating the NF-kB/PPAR $\gamma$ signaling pathway (Xiang et al., 2016). Apart from these, other Chinese herbal formulas including Liuhedan (Peng et al., 2013), Huoxueingyidecoction (Ji et al., 2016), Tongxiahuayu decoction (Fang et al., 2007), Yihuoqingyi decoction (Chen et al., 2011), Chaihusihuang decoction (Wang H. et al., 2009), Dachaihu decoction (Cheng et al., 2008), and Qingyichengqi decoction (Zhang et al., 2014) are also effective treatments for AP.

Despite the promising effects of Chinese herbal formulas, huge differences between TCM and modern medicine still push TCM away from mainstream medicine (Liu et al., 2016). There 
are several issues and challenges in current studies: First, the drawbacks of traditional decoctions and its administration are obvious, such as discommodiousness, unstable efficacy, and uncontrollable quality. Second, the data that harvested from rigorously stochastic, double-blind, placebo-controlled trials with multiple centers and large samples is insufficient (Qiong et al., 2005). Third, the interaction between the complex chemical and biological systems of AP remains ambiguous. Finally, the pharmacokinetics of these formulas and their interactions with other medications should be further evaluated (Yao et al., 2016b).

\section{PLEIOTROPIC NATURAL PRODUCTS}

Pleiotropic natural products derived from medicinal plants through extraction, separation, and purification have clear chemical structures that are different from Chinese herbal formulas and crude extracts (de Oliveira et al., 2015; Bulaj et al., 2016; Yao et al., 2016b). Pure natural products, as carriers of modern herbal medicine advanced technology, are considered as the only way for TCM to realize modernization and internationalization (Normile, 2003; Yao et al., 2016b). Natural products have been used in Japan since the 1970s and have been widely developed in Singapore and other places (Normile, 2003). In Europe and the United States, 25\% of prescriptions contain at least one extract from higher plants or compounds. In Germany, pure natural products are considered as drugs rather than food supplements and are covered by medical insurance (Yao et al., 2016a). In short, pure natural products in foreign countries have a good application base and a wide range of markets.

Pleiotropic natural products feature the following advantages: (1) well-known molecular weight, physical and chemical characteristics and easy qualitative and quantitative analyses; (2) various technique indexes can be controlled in the preparation process, and strict quality control can be respected; (3) the concentration of TCM effective ingredients can be greatly increased, enhancing medicine absorption in the body, thereby overcoming the fatal weakness of the slow effect of TCM; and (4) the pollution of harmful substances such as heavy metal ions and residual pesticide, can be maximally eliminated (Zhang et al., 2008b, 2009). Many pure natural products such as emodin, baicalin, resveratrol, and curcumin have been found to have significant therapeutic benefits against AP (Table 2). However, there is lack of clinical researches about the doses and side effects of these natural products at present.

\section{Emodin}

Emodin (1,3,8-trihydroxy-6-methylanthraquinone) (Figure 3A) is a natural anthraquinone derivative isolated from the roots and rhizomes of numerous plants, such as Rhamnaceae, Polygonum, Liliaceae, and Leguminosae, and especially, from the Chinese herb Rheum palmatum L., as an active compound, which has been utilized to treat critical illness (e.g., AP) in China for many years (Dong et al., 2016). Previous pharmacological studies have shown that emodin possesses biological activities such as purgative, anti-fibrotic, vasorelaxant, and immunosuppressive (Dong et al., 2016). Emodin also possesses an anti-inflammatory property in particular (Li et al., 2015). Early cell culture and animal research have revealed that emodin at the doses of $10-60 \mathrm{mg} / \mathrm{kg}$ could significantly reduce the mortality and have anti-AP effects (Wu et al., 2000, 2014; Ni et al., 2014a; Yao et al., 2015). Currently, studies suggest that emodin ameliorates AP through multiple targets; however, the exact mechanism underlying the effect of emodin in AP has not been completely addressed.

The paramount pathological manifestations of AP are pancreatic edema, hemorrhage, necrosis, and inflammatory infiltration, and increased pancreatic enzymes (Whitcomb, 2006). Emodin, as a direct NF- $\mathrm{BB}$ inhibitor, could result in an antioxidation response and subsequently suppress the expression of pro-inflammatory cytokines (Yao et al., 2015). Moreover, our previous research discovered 32 differentially expressed proteins from SAP rats with or without emodin treatment by using iTRAQ-based quantitative proteomic analysis, and a new biomarker, serine protease high-temperature requirement A1 (HTRA1), was found to associate with SAP. Further work found that one of the mechanisms underlying emodin against SAP involved inhibiting the HTRA1/TGF- $\beta 1 / \mathrm{NF}-\kappa \mathrm{B}$ signaling cascade and subsequent inflammatory responses (Li et al., 2016a).

Emodin can also promote cell apoptosis and reduce necrosis occurring in the pancreatic acinar cell or inflammatory cells, for example, emodin inhibits SIRS in SAP rats via inducing neutrophil apoptosis via the calcium-mediated caspase-12 signaling pathway (Ni et al., 2014b; Yin et al., 2016). In addition, the protective effects of emodin on ER stress responses are mediated by significantly attenuating calcium overload and decreasing the expression of ER chaperone immunoglobulinbinding protein (Bip), PERK, ATF6, IRE1 $\alpha$, TRAF2 and ASK1, as well as inhibiting the phosphorylation of MAPKs (Wu et al., 2013, 2014). Schmitt et al. (2004) reported that claudin and occludin expression in the pancreas are obviously reduced in AP, suggesting a possible role of tight junction disruption in interstitial edema formation. Emodin administration could increase claudin and occludin expression and reduce paracellular permeability in the pancreas (Xia et al., 2012). Furthermore, previous research indicated that emodin could reduce pancreatic ischemia and tissue injury through decreasing the expression of thromboxane-2 (TXB2) protein, which is a stable metabolite of TXA2 that has abilities to induce the deformation, release, and secretion of platelets (Kusterer et al., 1991; Wu et al., 2000). Interestingly, many studies have shown that the regeneration and repair of pancreatic injury occurs after AP (Konturek et al., 1997). Transforming growth factor- $\beta 1$ (TGF- $\beta 1$ ) is found to be involved in the pathogenesis of AP, especially pancreatic regeneration (Riesle et al., 1997). Emodin combined with somatostatin analogs may be beneficial for the up-regulation of TGF- $\beta 1$ and EGF expression, which contributes to pancreatic repair and regeneration (Gong et al., 2002). Moreover, results from Konturek et al. (1998) have demonstrated that significantly up-regulated expression of epidermal growth factor (EGF) may restrict the extent of initial tissue damage and then accelerate pancreatic regeneration and repair in AP.

Emodin has a protective effect in pancreatitis-associated secondary organ damage (Wang et al., 2007; Ning et al., 2009; Xia et al., 2010; Xu et al., 2016), which is both a common 
TABLE 2 | List of pure natural products derived from medicinal plants for the treatment of AP.

\begin{tabular}{|c|c|c|c|c|}
\hline Natural products & Main source & Models & Mechanisms & Reference \\
\hline Emodin & $\begin{array}{l}\text { Rheum palmatum L., Polygonum } \\
\text { cuspidatum, Polygonum multiflorum, } \\
\text { Aloe vera, Cassia obtusifolia, Radix et } \\
\text { Rhizoma Rhei }\end{array}$ & $\begin{array}{l}\text { SAP rats }(\mathrm{NaTc}) \\
\text { AP rats }(\mathrm{NaTc}) \\
\text { SAP rats }(\mathrm{NaTc}) \\
\text { AR42J cells (caerulein + LPS) } \\
\text { ANP rats }(\mathrm{NaTc}) \\
\text { SAP rats (NaTc) } \\
\text { AP rats (caerulein) } \\
\text { SAP rats neutrophils (NaTc) } \\
\text { SAP/SIRS rats pM } \Phi(\mathrm{NaTc})\end{array}$ & 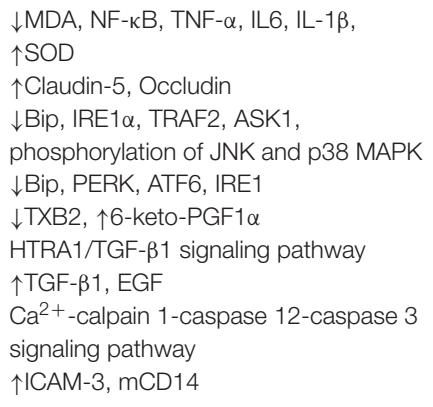 & $\begin{array}{l}\text { Yao et al., } 2015 \\
\text { Xia et al., } 2012 \\
\text { Wu et al., } 2013 \\
\text { Wu et al., } 2014 \\
\text { Wu et al., } 2000 \\
\text { Li et al., 2016a } \\
\text { Gong et al., } 2002 \\
\text { Yin et al., } 2016 \\
\text { Ni et al., 2014a,b }\end{array}$ \\
\hline \multirow[t]{6}{*}{ Baicalin } & \multirow[t]{6}{*}{ Scutellaria baicalensis Georgi } & AP rats (caerulein) & $\downarrow N F-\kappa B, T N F-\alpha$ & Xue et al., 2006 \\
\hline & & SAP rats (NaTc) & $\downarrow$ TNF- $\alpha$, IL-6, MDA, PLA2 & Zhang et al., 2007c \\
\hline & & SAP rats (NaTc) & $\downarrow N O, M D A, T N F-\alpha$ & Zhang et al., 2008b \\
\hline & & SAP rats (NaTc) & $\downarrow P$-selectin, TNF- $\alpha, \uparrow$ Caspase-3 & Xiping et al., 2009b \\
\hline & & SAP rats (NaTc) & $\downarrow \| L-1 \beta, P A F$, TXB2, PLA2, $P$ PE2 & Zhang et al., 2009b \\
\hline & & SAP rats (NaTc) & $\downarrow \mathrm{Bcl} 2, \uparrow \mathrm{Bax}$ & Xiping et al., 2009a \\
\hline \multirow[t]{3}{*}{ Resveratrol } & \multirow{3}{*}{$\begin{array}{l}\text { Grapes, berries, peanuts, soya beans, } \\
\text { red win, rhubarb, giant knotweed } \\
\text { rhizome, Eranthis hyemalis }\end{array}$} & $\begin{array}{l}\text { AP rats }(\mathrm{CCK} 8) \\
\mathrm{AP} \text { rats }(\mathrm{NaTc})\end{array}$ & $\begin{array}{l}\downarrow N F-\kappa B, T N F-\alpha, \uparrow C A T \text {, glutathione } \\
\downarrow N F-\kappa B, \text { AP- } 1, \text { TNF- } \alpha, \text { IL- } 6 \text {, iNOS }\end{array}$ & $\begin{array}{l}\text { Szabolcs et al., } 2006 \\
\text { Gulcubuk et al., } 2014\end{array}$ \\
\hline & & AP rats (caerulein) & $\downarrow I L-1 \beta$, MDA, $\uparrow I L 10$, GSH-Px, SOD & Carrasco et al., 2014 \\
\hline & & $\begin{array}{l}\text { SAP rats }(\mathrm{NaTc}) \\
\text { SAP rats }(\mathrm{NaTc}) \\
\text { SAP rats }(\mathrm{NaTc}) \text { pM } \Phi\end{array}$ & $\begin{array}{l}\downarrow \text { MDA, ICAM- } 1, \text { VCAM- } 1, \text { TNF- } \alpha, \\
\uparrow S O D \\
\downarrow P L A 2,\left[\mathrm{Ca}^{2+}\right] \mathrm{i}, \uparrow \mathrm{Ca}^{2+}-\mathrm{Mg}^{2+} \text {-ATPase, } \\
\mathrm{Ca}^{2+}-\mathrm{ATPase} \\
\downarrow N F-\kappa \mathrm{B}, \mathrm{NNOS}, \mathrm{TNF}-\alpha, \mathrm{IL}-1, \mathrm{NO}\end{array}$ & $\begin{array}{l}\text { Jha et al., } 2012 \\
\text { Wang et al., } 2008 \\
\text { Ma et al., } 2005\end{array}$ \\
\hline Dihydro-resveratrol & $\begin{array}{l}\text { Orchidaceae, Cannabis sativa L., A } \\
\text { metabolite of trans-resveratrol in the } \\
\text { human body }\end{array}$ & $\mathrm{AP}$ rats (caerulein) & $\begin{array}{l}\downarrow \text { MDA, NADPH oxidase, MPO, TNF- } \alpha \text {, } \\
\text { NF-кB, IкB degradation, AKT } \\
\text { phosphorylation, } \uparrow \text { glutathione, PIЗK }\end{array}$ & Tsang et al., 2016 \\
\hline \multirow[t]{5}{*}{ Curcumin } & \multirow[t]{5}{*}{ Turmeric (Curcuma longa) } & AP rats $(\mathrm{NaTc})$ & $\downarrow N F-\kappa B, A P-1$ & Gulcubuk et al., 2013 \\
\hline & & SAP rats (NaTc) & $\downarrow T L R 4, N F-\kappa B$ & Zhong, 2015 \\
\hline & & AP rats $(\mathrm{NaTc})$ & $\downarrow \mathrm{TNF}-\alpha$, IL6 & Gulcubuk et al., 2006 \\
\hline & & $\mathrm{AP}$ rats $(\mathrm{NaTc})$ & $\downarrow \mathrm{MDA}, \mathrm{NO}$, bacterial translocation & Gulcubuk et al., 2005 \\
\hline & & AP mice (caerulein) & $\uparrow P P A R \gamma, \downarrow N F-\kappa B, T N F-\alpha$ & Yu et al., 2011 \\
\hline Ligustrazine & $\begin{array}{l}\text { Ligusticum chuanxiong Hort., Curcuma } \\
\text { aromatica Salisb., Jatropha podagrica } \\
\text { Hook }\end{array}$ & $\begin{array}{l}\text { AP rats and acinar cells } \\
\text { (caerulein) }\end{array}$ & $\downarrow p 38$ Erk MAPK pathways & Chen et al., 2016 \\
\hline Honokiol & Magnolia officinalis Rehd. Et Wils & SAP mice (caerulein) & $\begin{array}{l}\downarrow T N F-\alpha, \text { IL1, NO, HMGB1, MPO } \\
\uparrow \text { elF2 } \alpha \text { phosphorylated, CHOP, } \\
\text { caspase-3 }\end{array}$ & Weng et al., 2012 \\
\hline
\end{tabular}

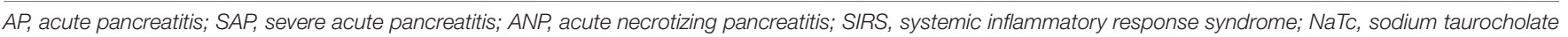

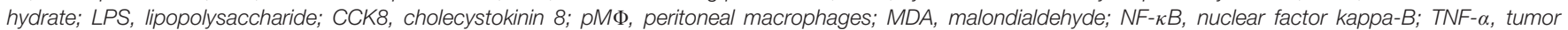

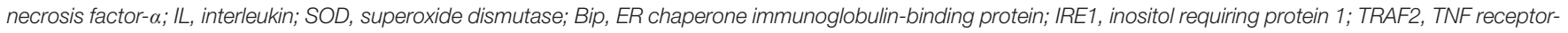

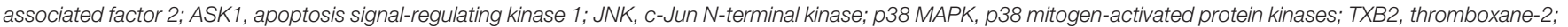

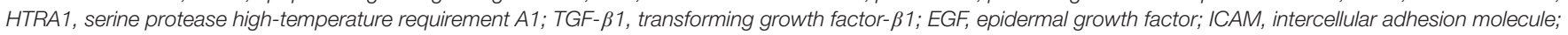

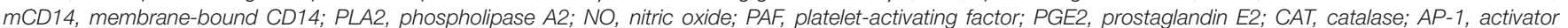

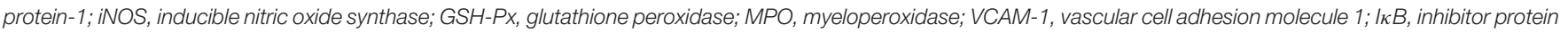

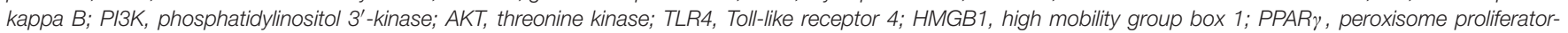
activated receptor gamma; CHOP, C/EBP homologous protein.

serious SAP complication and a main cause of death. Pancreatitisassociated lung injury occurs as a consequence of significant pulmonary hyperemia, edema, and inflammatory infiltration in the lung tissues. Although the strategies in prevention and treatments have been improved, ALI still causes more than $50 \%$ of deaths in SAP (Bhatia, 2002). Emodin attenuates pulmonary edema and enhances alveolar epithelial barrier function by up-regulating the expression of claudin-4, claudin-5, occludin, AQP1, and AQP5 in lung tissue samples from rats with SAP-induced ALI (Xia et al., 2010; Xu et al., 2016). Several 


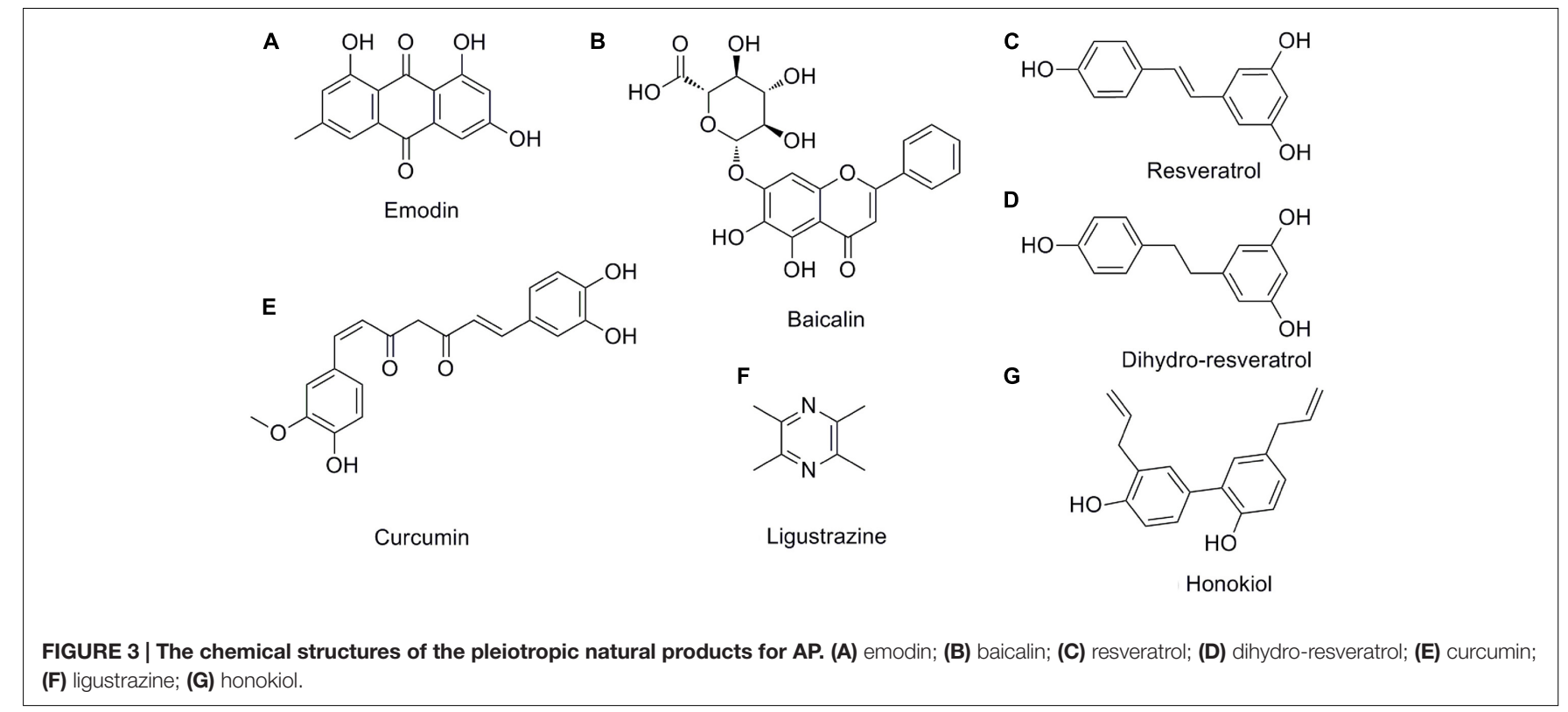

in vivo investigations have proposed that emodin can prevent the translocation of bacteria and endotoxins and promote the recovery of intestinal barrier function via inhibiting the intestinal mucosa cell apoptosis and up-regulating the serum leptin content (Ning et al., 2009). In addition, emodin-assisted early enteral nutrition (EAEEN) obviously abates the severity of secondary hepatic injury to function in the treatment of AP (Wang et al., 2007). However, emodin could also result in kidney toxicity, hepatotoxicity, and reproductive toxicity, particularly in longterm use with high-doses. Pharmacokinetic studies also indicated that emodin processed poor oral bioavailability because of its wide glucuronidation (Dong et al., 2016).

\section{Baicalin}

Baicalin (5,6,7-trihydroxyflavone-7-O-D-glucuronic acid) (Figure 3B) is one of the effective flavonoid compounds extracted from the dried root of Scutellaria baicalensis Georgi (Zhang et al., 2009a). In vitro experiments of baicalin demonstrated that it features multiple activities, such as resisting bacteria, antiinflammation, anti-oxidation, and inhibiting platelet aggregation, reducing endotoxin production and inducing apoptosis (Ueda et al., 2002; Shen et al., 2003). The pharmacological actions of baicalin are quite similar to those of octreotide, which is a somatostatin analog that can significantly inhibit pancreatic secretion (Shen et al., 2003; Tian et al., 2009). Fortunately, animal researches indicated that baicalin $(50-100 \mathrm{mg} / \mathrm{kg}$ ) has diverse pharmacological actions associated with antagonizing many stages of SAP onset and is much cheaper than octreotide, thus implying that baicalin is a promising medication for AP treatment. Early studies reported that baicalin could inhibit digestive enzyme activity, reduce pancreas necrosis, restrict lipid peroxidation, and increase the SAP rat survival rate (Zhang et al., 2007c, 2009a).

The beneficial effects of baicalin appear to be mediated by inhibiting NF- $\mathrm{KB}$ and TNF- $\alpha$ activity and increasing caspase-3 expression in multiple organs (Xue et al., 2006). Furthermore, baicalin may be applied for decreasing the expression levels of the P-selectin protein, which serves as a marker for leukocyteendothelial cell adhesion and active leukocyte-mediated organ injury and plays a key role in the progression of AP (Xiping et al., 2009b). Complementarily, early treatment with baicalin exerts significant protective effects on SAP-induced multiple organ injuries, such as liver (Zhang et al., 2008a), kidney (Zhang et al., 2007b), intestinal mucosa (Zhang et al., 2009a), heart (Xiping et al., 2007), and thymus (Xiping et al., 2010), with their possible mechanisms associated with inhibiting inflammatory mediators and inducing apoptosis.

\section{Resveratrol}

Resveratrol (trans-3,5, $4^{\prime}$-trihydroxystilbene) (Figure 3C), as a naturally polyphenolic phytoalexin, exists in almost 70 vegetables and fruits or food products, especially in grapes and red wine. It also has been identified as a major active ingredient in giant knotweed rhizome and rhubarb in TCM (Ma et al., 2011). Resveratrol is considered to be a good candidate for the treatment of oxidative and/or inflammatory diseases due to its antioxidant and anti-inflammatory activities and ease of extraction (Ma et al., 2011). Moreover, it has received an increasing amount of attention in treating AP (Meng et al., 2005b; Szabolcs et al., 2006). Previous animal studies have identified and documented the role of resveratrol (10-30 mg/ $/ \mathrm{kg})$ in decreasing the levels of amylase and lipase enzymes, mitigating histological damage in the pancreas and extra-pancreatic organs and reducing the mortality in the case of $\mathrm{AP}$, indicating the therapeutic effect of resveratrol against AP (Li et al., 2006; Wang et al., 2008).

Resveratrol can decrease the production and release of pro-inflammatory mediators, such as IL-1, IL-6, IL-8, TNF- $\alpha$, and NO, via blocking the activation of NF- $\mathrm{KB}$ and AP-1 and the associated kinases and increasing anti-inflammatory 
cytokine IL-10 levels (Ma et al., 2005; Szabolcs et al., 2006; Carrasco et al., 2014; Gulcubuk et al., 2014). At the same time, resveratrol suppressed the expression of intercellular adhesion molecule-1 (ICAM-1), which could mediate leukocyte adhesion to endothelium and reduce the infiltration of leukocytes into inflammatory sites (Meng et al., 2005b; Ma et al., 2011; Jha et al., 2012).

Leonard et al. (2003) demonstrated that resveratrol can clear hydroxyl superoxides and metal inductive radicals in AP as a high-efficiency scavenger. The results from recent studies also demonstrated that resveratrol exerts a protective effect against lipid peroxidation in the membrane and prevents DNA damage via the inhibition of the NF- $\mathrm{B}$ pathway and subsequent suppression of ROS products (Meng et al., 2005a; Li et al., 2006; Ma et al., 2011). In addition, it has been observed that SOD activity decreases, and the MDA activity significantly increases in organs in the early phases of AP; resveratrol reverses these phenomena to reduce the AP induced oxidative damage in the pancreas (Li et al., 2006).

Another advantage of resveratrol is that it also appears to reduce the intracellular calcium overload through restoring the intracellular calcium regulatory mechanisms, which limit not only pancreatic tissue injury but also secondary organ injury (Wang et al., 2008). Studies of SAP have shown that resveratrol may stabilize erythrocytes, improve the decrease of blood flow, decrease blood viscosity and leukocyte-endothelial interaction, thus decreasing thrombus formation and ameliorating the hypercoagulable state existing in every stage of SAP (Sha et al., 2013). Moreover, resveratrol could suppress microcirculatory disturbance via inhibition of the renin-angiotensin system (RAS) system and regulation of an unbalanced ET/NO status (Ma et al., 2011).

Resveratrol also has the ability to relieve injury in extrapancreatic organs in SAP such as intestines, liver, brain and lungs through the up-regulation of Bcl-2 and the down-regulation of Bax, cytochrome $c$, and caspase-3 levels (Meng et al., 2005b; Jha et al., 2008, 2009; Sha et al., 2008). In short, resveratrol has shown potential therapeutic effects in cases of AP by inhibiting the release of inflammatory mediators, promoting antioxidant effects, regulating cytoplasm calcium homeostasis, reversing microcirculatory disturbance, and inducing apoptosis.

When orally consumed, trans-resveratrol is rapidly metabolized in the human colon by gut bacteria and converted to dihydro-resveratrol (3,5,4'-trihydroxy bibenzyl), Figure 3D (Tsang et al., 2016). Importantly, the solubility of dihydroresveratrol was at least five times higher than trans-resveratrol while exhibiting a much lower cytotoxicity; thus, dihydroresveratrol is particularly suitable for patients unresponsive to trans-resveratrol due to the lack of proper microbial strains (Tsang et al., 2016). Lin et al. (2016) and Tsang et al. (2016) demonstrated that dihydro-resveratrol could significantly ameliorate pancreatic oxidative damage and AP-associated lung injury. The underlying molecular mechanisms involve in the decreased production of intracellular reactive oxidative products and pro-inflammatory cytokines, and the inhibition of the NF$\kappa \mathrm{B}$ and phosphatidylinositol $3^{\prime}$-kinase (PI3K)-serine/threonine kinase (AKT) signaling pathways.

\section{Curcumin}

Curcumin (diferuloylmethane, Figure 3E) is a turmeric polyphenol derived from Curcuma longa (turmeric), which has been prized in Ayurvedic medicine since ancient times for the treatment of inflammatory conditions due to its various pharmacological benefits including antioxidant and antiinflammatory properties (Jurenka, 2009; Shehzad et al., 2013). Based on the results of cell cultures, animal models and clinical trials, ample evidence validates that curcumin at the doses of $100 \mathrm{mg} / \mathrm{kg}$ may have potential as a therapeutic agent in AP (Gulcubuk et al., 2013; Zhong, 2015). Curcumin's improvement of AP is achieved through repressing the infiltration of inflammatory cells, inhibiting lipid peroxidation, and regulating TLR-4/NF- $\kappa$ B and PPAR $\gamma / N F-\kappa B$ signaling pathway, preventing free radical injury and the prevalence of bacterial translocation, thereby reducing trypsin activation, oxidative enzymes, and tissue injury (Gulcubuk et al., 2005, 2006, 2013; Yu et al., 2011; Zhong, 2015). Moreover, curcumin suggests that the activation of caspase- 3 may lead to an increase in apoptosis in the early and late phases of experimental AP.

\section{Others}

Ligustrazine (tetramethylpyrazine, Figure 3F) (Chen et al., 2016) and honokiol (3,5'-diallyl-4,2' -dihydroxybiphenyl, Figure 3G) (Weng et al., 2012) possess special pharmacological activities associated with the acceleration of acinar cell apoptosis at an early phase of AP. Thus, they may soon join the list of candidate drugs in the treatment of AP besides the above-mentioned natural products. However, in-depth research is necessary to confirm this hypothesis.

\section{NETWORK TARGET PREDICTION}

Traditional Chinese medicine is one of the promising strategies in treating AP due to its multi-targeting and lower side effects (Yao et al., 2016a). Despite massive investments in TCM research and development, there has been no significant increase in the number of new drugs approved or translated for clinical use. Single targeted drug discovery has proved to be ineffective in combating complex diseases that harbor robust biological networks such as AP. Network pharmacology prediction provides a highly useful method for drug targets, which differs from conventional single target intervention by integrating network biology and polypharmacology, thereby exploring drugs to target numerous proteins or networks involved in a disease (Cheng et al., 2015; Gao et al., 2016). In this review, we also discuss the application of network pharmacology for AP innovative drugs discovery.

We searched the TCMID, DrugBank, GeneCards, and STITCH databases to validate 68 AP targets based on six pure natural products (emodin, baicalin, resveratrol, curcumin, ligustrazine, and honokiol) mentioned above. Subsequently, a compound-target network was constructed using Cytoscape 3.3.0 software (Cheng et al., 2015; Xiang et al., 2016). As shown in Figures 4A-F, in this network, red rectangles and different colors-ellipses, respectively, correspond to natural products and 

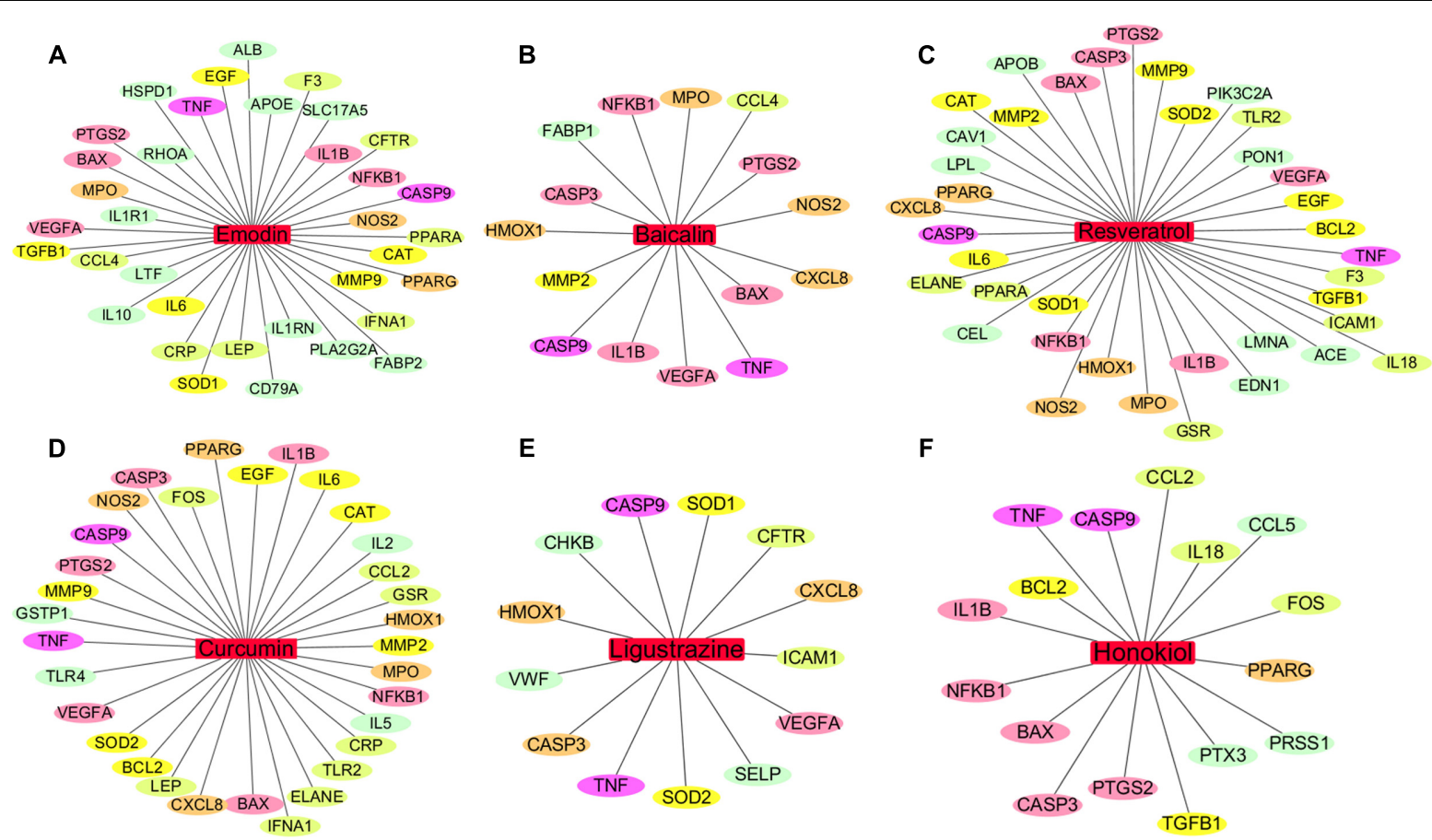

$\mathbf{F}$

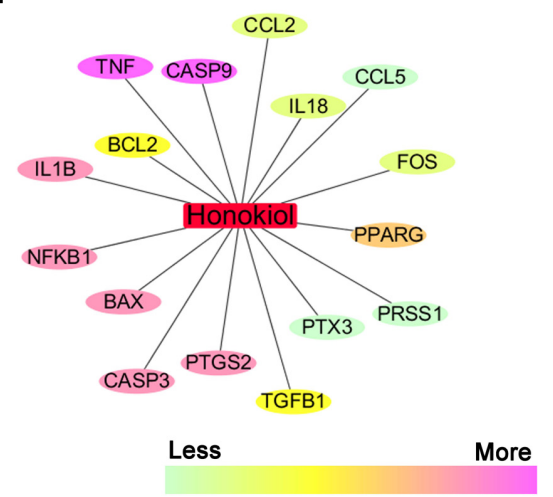

FIGURE 4 | The natural product-target network predicted by network pharmacology. (A-F) In this network, red rectangles correspond to natural products (emodin, baicalin, resveratrol, curcumin, ligustrazine, and honokiol), and different colors-ellipses correspond to targets. The colors of each target transit from green to purple, indicating its increased importance in the process of these natural products for AP treatment. In the relationships between natural products and targets, resveratrol corresponds to the highest number of candidate targets (degree $=38$ ), followed by emodin (degree $=35)$, curcumin $($ degree $=32)$, honokiol (degree $=16$ ), baicalin (degree $=15$ ), and ligustrazine (degree $=13$ ). The candidate targets in the progression of AP mainly related to inflammation (TNF, NFKB1, PTGS2, IL1B, IL6, IL10, etc.), apoptosis (CASP9, CASP3, BAX, BCL2, etc.), oxidation-reduction (NOS2, SOD1, MPO, CAT, etc.), and lipid metabolic process (PPARA, PPARG, APOE, APOB, etc.), as well as the maintenance of $\mathrm{Ca}^{2+}$ homeostasis (ELANE, CAV1, CCL4, etc.) and microcirculation (IFNA1, SELP, etc.).

targets, and the colors of each target transit from green to purple, indicating its increased importance in the process of these natural products for AP treatment. In the relationships between natural products and targets, resveratrol corresponds to the highest number of candidate targets (degree $=38$ ), followed by emodin (degree $=35)$, curcumin $($ degree $=32$ ), honokiol (degree $=16)$, baicalin $($ degree $=15)$, and ligustrazine (degree $=13)$.

These emphasize the integral function of natural products in the treatment of AP through providing multiple therapeutic effects on multiple targets as compared with Western medicine, which usually focuses on a single target. The candidate targets in the progression of AP mainly related to inflammation (TNF, NFKB1, PTGS2, IL1B, IL6, IL10, etc.), apoptosis (CASP9, CASP3, BAX, BCL2, etc.), oxidation-reduction (NOS2, SOD1, MPO, CAT, etc.), and lipid metabolic process (PPARA, PPARG, APOE, APOB, etc.), as well as the maintenance of $\mathrm{Ca}^{2+}$ homeostasis (ELANE, CAV1, CCL4, etc.) and microcirculation (IFNA1, SELP, etc.). Especially, inflammation and apoptosis-related targets (e.g., TNF, CASP9, NFKB1, BAX, IL1B, CASP3) show the higher correlation (degree $=6$ or 5 ), demonstrating the potential therapeutic effect of these natural products on AP through modulating these relevant proteins. Except for the common targets, each natural product has specific regulation on some targets. For instance, resveratrol has a potential regulatory effect on CAV1 that promotes the maintenance of intracellular calcium homeostasis; emodin maybe benefit to regulate blood lipid levels in AP by regulating the expression and function of APOE that contributes to the lipoprotein biosynthetic, catabolic, and metabolic process; ligustrazine may improve the state of hypercoagulability and microcirculation during AP via regulating VWF and SELP expression. Each natural product acts on multiple targets, and the different natural products share the synergistic targets, which is the basis of action of pure natural products. However, since the compound-target network is only obtained by data mining combined with computer simulation predictions, whether the above natural products against AP through these potential targets still require further experimental validation.

\section{POTENTIAL TOXIC NATURAL PRODUCTS FOR PANCREAS}

Recent research pointed out an increasing interest concerning the health benefits of natural products, and they have been 


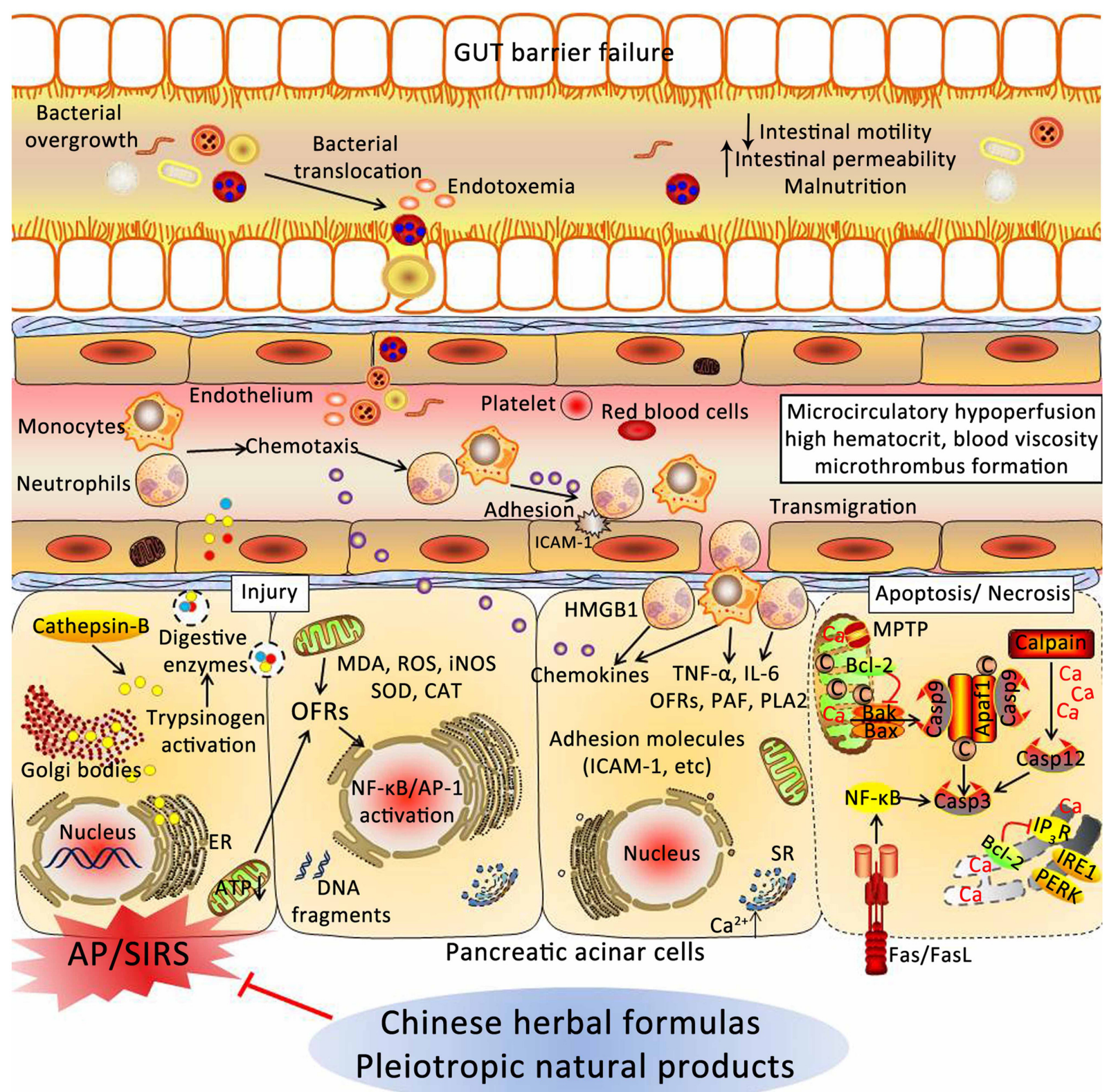

FIGURE 5 | Underlying mechanisms of Chinese herbal medicines (CHMs) for the treatment of AP. CHMs may prevent cellular damage in pancreas associated with AP through a variety of mechanisms, including: (1) depressing the synthesis and secretion of digestive enzymes; (2) depressing oxidative stress through increased antioxidant levels and decreased the excessive OFRs; (3) inhibiting activation of inflammatory pathways; (4) relieving ER stress via PERK and IRE1; (5) restoring the intracellular calcium regulatory mechanisms; (6) inducing the switch from apoptosis to necrosis in pancreatic cells; (7) increasing pancreatic blood flow, and reducing blood viscosity; and (8) restoring intestinal barrier function and blocking bacterial translocation. AP, acute pancreatitis; SIRS, systemic inflammatory response syndrome; ER, endoplasmic reticulum; ATP, adenosine triphosphate; OFRs, oxygen free radicals; MDA, malondialdehyde; ROS, reactive oxygen species; iNOS, inducible nitric oxide synthase; SOD, superoxide dismutase; CAT, catalase; NF-кB, nuclear factor kappa-B; AP-1, activator protein-1; HMGB1, high mobility group box 1; TNF- $\alpha$, tumor necrosis factor- $\alpha$; IL-6, interleukin-6; PAF, platelet-activating factor; PLA2, phospholipase A2; ICAM-1, intercellular adhesion molecule-1; SR, sarcoplasmic reticulum; MPTP, mitochondrial permeability transition pore; IP3R, inositol 1,4,5-trisphosphate receptor; PERK, RNA-activated protein kinase-like ER kinase; IRE1, inositol requiring protein 1; Apaf-1, apoptotic protease activating factor-1.

considered a good complementary and alternative medicine in the treatment of AP. As a coin has two sides, exaggerating the safety and non-toxic advantages of natural products leads to the negligence of potential pancreas to toxicity for a long time. There are very limited data of about the potential toxicity of herbs or natural products on the pancreas. Currently, three case reports showed a probable AP induced by saw-palmetto, a phytotherapeutic agent for symptoms related to benign prostatic 
hyperplasia $(\mathrm{BPH})$. It has been postulated that saw-palmetto stimulates estrogenic receptors and then increases triglyceride levels or induces a hypercoagulable state that leads to pancreatic necrosis (Jibrin et al., 2006; Wargo et al., 2010; Bruminhent et al., 2011). The evidence derived mainly from random case reports described possible Ceramium kondoi or horsetail infusions induced AP (Kim et al., 2013; Garcia Gavilan et al., 2017). Experimental results indicate that 1-cyano-2-hydroxy-3-butene (CHB), a nitrile derived from many cruciferous plants, is a selective pancreatotoxin. $\mathrm{CHB}$ is also a possible inducer of tissue glutathione in the pancreas, even at toxic doses $(200 \mathrm{mg} \mathrm{CHB} / \mathrm{kg}$ body weight) (Wallig et al., 1988). L-canavanine extracting from Hedysarum alpinum seeds processes potential pancreatotoxicity when it's severs as an antitumor. Histological researches of tissues from rats treated with canavanine $(3.0 \mathrm{~g} / \mathrm{kg})$ for 6 days revealed pancreatic acinar cell atrophy and fibrosis; and serum amylase and lipase levels were increased after one sc injection of $2.0 \mathrm{~g} / \mathrm{kg}$ canavanine (Thomas and Rosenthal, 1987). Overall, natural products have the potential to be not only beneficial but also harmful under a number of medical conditions. This information should prompt clinicians to consider natural product a potential cause of AP.

\section{CONCLUSION}

Acute pancreatitis is the leading cause of hospital admission for gastrointestinal diseases, and the effective strategies are

\section{REFERENCES}

Baggaley, E. M., Elliott, A. C., and Bruce, J. I. (2008). Oxidant-induced inhibition of the plasma membrane $\mathrm{Ca}^{2+}$-ATPase in pancreatic acinar cells: role of the mitochondria. Am. J. Physiol. Cell Physiol. 295, C1247-C1260. doi: 10.1152/ ajpcell.00083.2008

Banks, P. A., Bollen, T. L., Dervenis, C., Gooszen, H. G., Johnson, C. D., Sarr, M. G., et al. (2013). Classification of acute pancreatitis-2012: revision of the Atlanta classification and definitions by international consensus. Gut 62, 102-111. doi: 10.1136/gutjnl-2012-302779

Bhatia, M. (2002). Novel therapeutic targets for acute pancreatitis and associated multiple organ dysfunction syndrome. Curr. Drug Targets Inflamm. Allergy 1, 343-351. doi: 10.2174/1568010023344517

Binker, M. G., Richards, D., Gaisano, H. Y., and Cosen-Binker, L. I. (2015). ER stress-associated CTRC mutants decrease stimulated pancreatic zymogen secretion through SIRT2-mediated microtubule dysregulation. Biochem. Biophys. Res. Commun. 463, 329-335. doi: 10.1016/j.bbrc.2015.05.064

Bruce, J. I., and Elliott, A. C. (2007). Oxidant-impaired intracellular $\mathrm{Ca}^{2+}$ signaling in pancreatic acinar cells: role of the plasma membrane $\mathrm{Ca}^{2+}$-ATPase. Am. J. Physiol. Cell Physiol. 293, C938-C950. doi: 10.1152/ajpcell.00582.2006

Bruminhent, J., Carrera, P., Li, Z., Amankona, R., and Roberts, I. M. (2011). Acute pancreatitis with saw palmetto use: a case report. J. Med. Case Rep. 5:414. doi: 10.1186/1752-1947-5-414

Bulaj, G., Ahern, M. M., Kuhn, A., Judkins, Z. S., Bowen, R. C., and Chen, Y. (2016). Incorporating natural products, pharmaceutical drugs, self-care and digital/mobile health technologies into molecular-behavioral combination therapies for chronic diseases. Curr. Clin. Pharmacol. 11, 128-145. doi: 10.2174/ 1574884711666160603012237

Camello-Almaraz, M. C., Pozo, M. J., Murphy, M. P., and Camello, P. J. (2006). Mitochondrial production of oxidants is necessary for physiological calcium oscillations. J. Cell. Physiol. 206, 487-494. doi: 10.1002/jcp.20498

Carrasco, C., Holguin-Arevalo, M. S., Martin-Partido, G., Rodriguez, A. B., and Pariente, J. A. (2014). Chemopreventive effects of resveratrol in a rat model limited. Thus, it is urgent to search ways to prevent and treat AP. In this review, experimental evidence implicates CHMs in the prevention and treatment of AP through various underlying mechanisms, including: (1) depressing the synthesis and secretion of digestive enzymes; (2) depressing oxidative stress through increased antioxidant levels and decreased the excessive OFRs; (3) inhibiting activation of inflammatory pathways; (4) relieving ER stress via PERK and IRE1; (5) restoring the intracellular calcium regulatory mechanisms; (6) inducing the switch from apoptosis to necrosis in pancreatic cells; (7) increasing pancreatic blood flow, and reducing blood viscosity; and (8) restoring intestinal barrier function and blocking bacterial translocation (Figure 5). However, more convincing studies are needed to confirm this hypothesis.

\section{AUTHOR CONTRIBUTIONS}

HX, QZ, BQ, XT, SX, HS, JQ, and DS wrote the manuscript.

\section{FUNDING}

This work was financially supported by the National Natural Science Foundation of China (No. 81373875) and the Key Project Supported by Clinical Ability Construction of Liaoning Province (No. LNCCC-A03-2015).

of cerulein-induced acute pancreatitis. Mol. Cell. Biochem. 387, 217-225. doi: 10.1007/s11010-013-1887-0

Chen, H., Li, F., Jia, J. G., Diao, Y. P., Li, Z. X., and Sun, J. B. (2010). Effects of traditional Chinese medicine on intestinal mucosal permeability in early phase of severe acute pancreatitis. Chin. Med. J. 123, 1537-1542.

Chen, J., Chen, J., Wang, X., Wang, C., Cao, W., Zhao, Y., et al. (2016). Ligustrazine alleviates acute pancreatitis by accelerating acinar cell apoptosis at early phase via the suppression of p38 and Erk MAPK pathways. Biomed. Pharmacother. 82, 1-7. doi: 10.1016/j.biopha.2016.04.048

Chen, Y. F., Sha, J. P., and Wu, Z. M. (2011). Synergetic effect of yihuo qingyi decoction (see text) and recombinant staphylokinase in treatment of severe acute pancreatitis of rats. J. Tradit. Chin. Med. 31, 103-106. doi: 10.1016/S02546272(11)60021-6

Chen, Z., Chen, Y., Pan, L., Li, H., Tu, J., Liu, C., et al. (2015). Dachengqi decoction attenuates inflammatory response via inhibiting HMGB1 mediated NF-kappaB and P38 MAPK signaling pathways in severe acute pancreatitis. Cell Physiol. Biochem. 37, 1379-1389. doi: 10.1159/000430403

Cheng, L., Pan, G. F., Zhang, X. D., Wang, J. L., Wang, W. D., Zhang, J. Y., et al. (2015). Yindanxinnaotong, a Chinese compound medicine, synergistically attenuates atherosclerosis progress. Sci. Rep. 5:12333. doi: 10.1038/srep 12333

Cheng, Y. X., Wang, M., and Cheng, X. (2008). Effect of dachaihu decoction in treating acute mild pancreatitis of Gan-qi stagnant type. Zhongguo Zhong Xi Yi Jie He Za Zhi 28, 793-796.

Chvanov, M., Petersen, O. H., and Tepikin, A. (2005). Free radicals and the pancreatic acinar cells: role in physiology and pathology. Philos. Trans. R. Soc. Lond. B Biol. Sci. 360, 2273-2284. doi: 10.1098/rstb.2005.1757

Criddle, D. N. (2016). Reactive oxygen species, $\mathrm{Ca}^{(2+)}$ stores and acute pancreatitis; a step closer to therapy? Cell Calcium 60, 180-189. doi: 10.1016/ j.ceca.2016.04.007

Dawra, R., Sah, R. P., Dudeja, V., Rishi, L., Talukdar, R., Garg, P., et al. (2011). Intra-acinar trypsinogen activation mediates early stages of pancreatic injury but not inflammation in mice with acute pancreatitis. Gastroenterology 141, 2210-2217.e2 doi: 10.1053/j.gastro.2011.08.033 
de Oliveira, M. R., Nabavi, S. F., Habtemariam, S., Erdogan Orhan, I., Daglia, M., and Nabavi, S. M. (2015). The effects of baicalein and baicalin on mitochondrial function and dynamics: a review. Pharmacol. Res. 100, 296-308. doi: 10.1016/j. phrs.2015.08.021

Deng, L. H., Yang, X. N., Huang, L., Xiang, D. K., and Xia, Q. (2008a). Effects of Chaiqinchengqi decoction on exocrine function of pancreatic acinar cells of acute pancreatitis rats. Sichuan Da Xue Xue Bao Yi Xue Ban 39, 555-557.

Deng, L. H., Yang, X. N., and Xia, Q. (2008b). Protective effects of Chaiqin Chengqi decoction on isolated pancreatic acinar cells in acute pancreatitis rats and the mechanisms. Zhong Xi Yi Jie He Xue Bao 6, 176-179.

Dong, X., Fu, J., Yin, X., Cao, S., Li, X., Lin, L., et al. (2016). Emodin: a review of its pharmacology, toxicity and pharmacokinetics. Phytother. Res. 30, 1207-1218. doi: $10.1002 /$ ptr.5631

Fang, B. J., Gao, P. Y., He, S. H., Chen, H., Shen, P., Zhang, Y. Y., et al. (2007). Clinical early intervention of Tongxia Huayu decoction on pancreatic microcirculatory disturbance in severe acute pancreatitis. Zhong Xi Yi Jie He Xue Bao 5, 134-136. doi: 10.3736/jcim20070206

Fu, Q., Qin, T., Chen, L., Liu, C. J., Zhang, X., Wang, Y. Z., et al. (2016). miR-29a up-regulation in AR42J cells contributes to apoptosis via targeting TNFRSF1A gene. World J. Gastroenterol. 22, 4881-4890. doi: 10.3748/wjg.v22.i20.4881

Gao, L., Wang, X. D., Niu, Y. Y., Duan, D. D., Yang, X., Hao, J., et al. (2016). Molecular targets of Chinese herbs: a clinical study of hepatoma based on network pharmacology. Sci. Rep. 6:24944. doi: 10.1038/srep24944

Garcia Gavilan, M. D., Moreno Garcia, A. M., Rosales Zabal, J. M., Navarro Jarabo, J. M., and Sanchez Cantos, A. (2017). Case of drug-induced acute pancreatitis produced by horsetail infusions. Rev. Esp. Enferm. Dig. 109, 301-304. doi: 10.17235/reed.2017.4157/2015

Gerasimenko, J. V., Gerasimenko, O. V., and Petersen, O. H. (2014). The role of $\mathrm{Ca}^{2+}$ in the pathophysiology of pancreatitis. J. Physiol. 592, 269-280. doi: 10.1113/jphysiol.2013.261784

Gillies, N., Pendharkar, S. A., Asrani, V. M., Mathew, J., Windsor, J. A., and Petrov, M. S. (2016). Interleukin-6 is associated with chronic hyperglycemia and insulin resistance in patients after acute pancreatitis. Pancreatology 16, 748-755. doi: 10.1016/j.pan.2016.06.661

Gong, Z., Yuan, Y., Lou, K., Tu, S., Zhai, Z., and Xu, J. (2002). Mechanisms of Chinese herb emodin and somatostatin analogs on pancreatic regeneration in acute pancreatitis in rats. Pancreas 25, 154-160. doi: 10.1097/00006676200208000-00007

Gulcubuk, A., Altunatmaz, K., Sonmez, K., Haktanir-Yatkin, D., Uzun, H., Gurel, A., et al. (2006). Effects of curcumin on tumour necrosis factor-alpha and interleukin-6 in the late phase of experimental acute pancreatitis. J. Vet. Med. A Physiol. Pathol. Clin. Med. 53, 49-54. doi: 10.1111/j.1439-0442.2006. 00786.x

Gulcubuk, A., Haktanir, D., Cakiris, A., Ustek, D., Guzel, O., Erturk, M., et al. (2013). Effects of curcumin on proinflammatory cytokines and tissue injury in the early and late phases of experimental acute pancreatitis. Pancreatology 13, 347-354. doi: 10.1016/j.pan.2013.05.005

Gulcubuk, A., Haktanir, D., Cakiris, A., Ustek, D., Guzel, O., Erturk, M., et al. (2014). The effects of resveratrol on tissue injury, oxidative damage, and pro-inflammatory cytokines in an experimental model of acute pancreatitis. J. Physiol. Biochem. 70, 397-406. doi: 10.1007/s13105-014-0317-4

Gulcubuk, A., Sonmez, K., Gurel, A., Altunatmaz, K., Gurler, N., Aydin, S., et al. (2005). Pathologic alterations detected in acute pancreatitis induced by sodium taurocholate in rats and therapeutic effects of curcumin, ciprofloxacin and metronidazole combination. Pancreatology 5, 345-353. doi: 10.1159/0000 86534

Guo, J., Jin, T., Lin, Z. Q., Wang, X. X., Yang, X. N., Xia, Q., et al. (2015). Effect of Chaiqin Chengqi decoction on cholecystokinin receptor 1-mediated signal transduction of pancreatic acinar cells in acute necrotizing pancreatitis rats. Chin. J. Integr. Med. 21, 29-35. doi: 10.1007/s11655-014-1750-5

Guo, J., Xue, P., Yang, X. N., Lin, Z. Q., Chen, Y., Jin, T., et al. (2013). The effect of Chaiqin Chengqi decoction () on modulating serum matrix metalloproteinase 9 in patients with severe acute pancreatitis. Chin. J. Integr. Med. 19, 913-917. doi: 10.1007/s11655-013-1653-x

Guo, Z. Z., Wang, P., Yi, Z. H., Huang, Z. Y., and Tang, C. W. (2014). The crosstalk between gut inflammation and gastrointestinal disorders during acute pancreatitis. Curr. Pharm. Des. 20, 1051-1062. doi: 10.2174/ 13816128113199990414
Hanna, E. M., Hamp, T. J., McKillop, I. H., Bahrani-Mougeot, F., Martinie, J. B., Horton, J. M., et al. (2014). Comparison of culture and molecular techniques for microbial community characterization in infected necrotizing pancreatitis. J. Surg. Res. 191, 362-369. doi: 10.1016/j.jss.2014.05.003

Hayden, M. S., and Ghosh, S. (2008). Shared principles in NF-kappaB signaling. Cell 132, 344-362. doi: 10.1016/j.cell.2008.01.020

Hirota, M., Ohmuraya, M., and Baba, H. (2006). The role of trypsin, trypsin inhibitor, and trypsin receptor in the onset and aggravation of pancreatitis. J. Gastroenterol. 41, 832-836. doi: 10.1007/s00535-006-1874-2

Hofbauer, B., Saluja, A. K., Lerch, M. M., Bhagat, L., Bhatia, M., Lee, H. S., et al. (1998). Intra-acinar cell activation of trypsinogen during caerulein-induced pancreatitis in rats. Am. J. Physiol. 275(2 Pt 1), G352-G362.

Jakkampudi, A., Jangala, R., Reddy, B. R., Mitnala, S., Nageshwar Reddy, D., and Talukdar, R. (2016). NF-kappaB in acute pancreatitis: mechanisms and therapeutic potential. Pancreatology 16, 477-488. doi: 10.1016/j.pan.2016. 05.001

Jha, R. K., Ma, Q., Lei, Z., and Sha, H. (2012). Resveratrol ameliorates the deleterious effect of severe acute pancreatitis. Cell Biochem. Biophys. 62, 397-402. doi: 10.1007/s12013-011-9313-2

Jha, R. K., Ma, Q., Sha, H., and Palikhe, M. (2009). Protective effect of resveratrol in severe acute pancreatitis-induced brain injury. Pancreas 38, 947-953. doi: 10.1097/MPA.0b013e3181b2bc54

Jha, R. K., Yong, M. Q., and Chen, S. H. (2008). The protective effect of resveratrol on the intestinal mucosal barrier in rats with severe acute pancreatitis. Med. Sci. Monit. 14, Br14-Br19.

Ji, C. H., Tang, C. W., Feng, W. M., Bao, Y., and Yao, L. Q. (2016). A Chinese Herbal decoction, Huoxue Qingyi decoction, promotes rehabilitation of patients with severe acute pancreatitis: a retrospective study. Evid. Based Complement. Alternat. Med. 2016, 3456510. doi: 10.1155/2016/3456510

Jia, G., Xiaoxiang, W., Ruijie, L., Xiaoxin, Z., Xiaonan, Y., Qing, X., et al. (2015). Effect of Chaiqinchengqi decoction on inositol requiring enzyme 1alpha in alveolar macrophages of dogs with acute necrotising pancreatitis induced by sodium taurocholate. J. Tradit. Chin. Med. 35, 434-439. doi: 10.1016/S02546272(15)30121-7

Jiang, C. G. (2010). The treatment of acute pancreatitis with Dachengqi decoction in 32 cases. Zhongguo Wei Zhong Bing Ji Jiu Yi Xue. 22, 249.

Jibrin, I., Erinle, A., Saidi, A., and Aliyu, Z. Y. (2006). Saw palmetto-induced pancreatitis. South. Med. J. 99, 611-612. doi: 10.1097/01.smj.0000215642. 76198.44

Jin, X., Feng, L., Hu, Y. Y., Tao, K., and Zhao, G. H. (2013). Effects and significance of electrical stimulation of the vagus nerve on plasma levels of nitric oxide and endothelin in severe acute pancreatitis in rats. J. Hepatobiliary Surg. 21, 62-64.

Jurenka, J. S. (2009). Anti-inflammatory properties of curcumin, a major constituent of Curcuma longa: a review of preclinical and clinical research. Altern. Med. Rev. 14, 141-153.

Kennedy, J. I., Askelund, K. J., Premkumar, R., Phillips, A. R., Murphy, R., Windsor, J. A., et al. (2016). Leptin is associated with persistence of hyperglycemia in acute pancreatitis: a prospective clinical study. Medicine 95:e2382. doi: 10.1097/md. 0000000000002382

Kim, D. B., Cho, Y. K., Song, H. J., and Song, B. C. (2013). A case of acute pancreatitis and acute hepatitis caused by ingestion of Ceramium kondoi. Korean J. Gastroenterol. 62, 306-309. doi: 10.4166/kjg.2013.62.5.306

Kim, M. S., Hong, J. H., Li, Q., Shin, D. M., Abramowitz, J., Birnbaumer, L., et al. (2009). Deletion of TRPC3 in mice reduces store-operated $\mathrm{Ca}^{2+}$ influx and the severity of acute pancreatitis. Gastroenterology 137, 1509-1517. doi: 10.1053/j.gastro.2009.07.042

Kishimoto, W., Nakao, A., Nakano, M., Takahashi, A., Inaba, H., and Takagi, H. (1995). Detection of superoxide free radicals in rats with acute pancreatitis. Pancreas 11, 122-126. doi: 10.1097/00006676-199508000-00002

Koh, Y. H., Tamizhselvi, R., and Bhatia, M. (2010). Extracellular signal-regulated kinase $1 / 2$ and c-Jun NH2-terminal kinase, through nuclear factor-kappaB and activator protein-1, contribute to caerulein-induced expression of substance $\mathrm{P}$ and neurokinin-1 receptors in pancreatic acinar cells. J. Pharmacol. Exp. Ther. 332, 940-948. doi: 10.1124/jpet.109.160416

Kolaczkowska, E., and Kubes, P. (2013). Neutrophil recruitment and function in health and inflammation. Nat. Rev. Immunol. 13, 159-175. doi: 10.1038/nri3399 Konturek, P. C., Dembinski, A., Warzecha, Z., Ceranowicz, P., Konturek, S. J., Stachura, J., et al. (1997). Expression of transforming growth factor-beta 1 and 
epidermal growth factor in caerulein- induced pancreatitis in rat. J. Physiol. Pharmacol. 48, 59-72.

Konturek, P. C., Dembinski, A., Warzecha, Z., Ihlm, A., Ceranowicz, P., Konturek, S. J., et al. (1998). Comparison of epidermal growth factor and transforming growth factor-betal expression in hormone-induced acute pancreatitis in rats. Digestion 59, 110-119. doi: 10.1159/000007483

Kruger, B., Albrecht, E., and Lerch, M. M. (2000). The role of intracellular calcium signaling in premature protease activation and the onset of pancreatitis. Am. J. Pathol. 157, 43-50. doi: 10.1016/s0002-9440(10)64515-4

Kubisch, C. H., and Logsdon, C. D. (2007). Secretagogues differentially activate endoplasmic reticulum stress responses in pancreatic acinar cells. Am. J. Physiol. Gastrointest. Liver Physiol. 292, G1804-G1812. doi: 10.1152/ajpgi.00078.2007

Kubisch, C. H., and Logsdon, C. D. (2008). Endoplasmic reticulum stress and the pancreatic acinar cell. Expert Rev. Gastroenterol. Hepatol. 2, 249-260. doi: 10.1586/17474124.2.2.249

Kusterer, K., Enghofer, M., Zendler, S., Blochle, C., and Usadel, K. H. (1991). Microcirculatory changes in sodium taurocholate-induced pancreatitis in rats. Am. J. Physiol. 260(2 Pt 1), G346-G351.

Lankisch, P. G., Apte, M., and Banks, P. A. (2015). Acute pancreatitis. Lancet 386, 85-96. doi: 10.1016/s0140-6736(14)60649-8

Lankisch, P. G., Lowenfels, A. B., and Maisonneuve, P. (2002). What is the risk of alcoholic pancreatitis in heavy drinkers? Pancreas 25, 411-412. doi: 10.1097/ 00006676-200211000-00015

Leonard, S. S., Xia, C., Jiang, B. H., Stinefelt, B., Klandorf, H., Harris, G. K., et al. (2003). Resveratrol scavenges reactive oxygen species and effects radicalinduced cellular responses. Biochem. Biophys. Res. Commun. 309, 1017-1026. doi: 10.1016/j.bbrc.2003.08.105

Lerch, M. M., and Gorelick, F. S. (2000). Early trypsinogen activation in acute pancreatitis. Med. Clin. North Am. 84, 549-563. doi: 10.1016/S0025-7125(05) 70239-X

Leveau, P., Wang, X., Soltesz, V., Ihse, I., and Andersson, R. (1996). Alterations in intestinal motility and microflora in experimental acute pancreatitis. Int. J. Pancreatol. 20, 119-125.

Li, G., Sun, C., Wu, G., Shi, F., Liu, A., and Yang, N. (2016a). iTRAQ-based quantitative proteomics identifies potential regulatory proteins involved in chicken eggshell brownness. PLoS ONE 11:e0168750. doi: 10.1371/journal.pone. 0168750

Li, G., Wu, X., Yang, L., He, Y., Liu, Y., Jin, X., et al. (2016b). TLR4-mediated NFkappaB signaling pathway mediates HMGB1-induced pancreatic injury in mice with severe acute pancreatitis. Int. J. Mol. Med. 37, 99-107. doi: 10.3892/ijmm. 2015.2410

Li, Y., Xiong, W., Yang, J., Zhong, J., Zhang, L., Zheng, J., et al. (2015). Attenuation of inflammation by emodin in lipopolysaccharide-induced acute kidney injury via inhibition of toll-like receptor 2 signal pathway. Iran. J. Kidney Dis. 9, 202-208.

Li, Y. H., Huang, Z. W., Xue, P., Guo, J., He, F. Q., You, Z., et al. (2008). Effects of Chaiqin Chengqi decoction on activation of nuclear factor-kappaB in pancreas of rats with acute necrotizing pancreatitis. Zhong Xi Yi Jie He Xue Bao 6, 180-184. doi: 10.3736/jcim20080215

Li, Z. D., Ma, Q. Y., and Wang, C. A. (2006). Effect of resveratrol on pancreatic oxygen free radicals in rats with severe acute pancreatitis. World J. Gastroenterol. 12, 137-140. doi: 10.3748/wjg.v12.i1.137

Lin, Z., Guo, J., Xue, P., Huang, L., Deng, L., Yang, X., et al. (2014). Chaiqinchengqi decoction regulates necrosis-apoptosis via regulating the release of mitochondrial cytochrome $c$ and caspase- 3 in rats with acute necrotizing pancreatitis. J. Tradit. Chin. Med. 34, 178-183. doi: 10.1016/S02546272(14)60075-3

Lin, Z. S., Ku, C. F., Guan, Y. F., Xiao, H. T., Shi, X. K., Wang, H. Q., et al. (2016). Dihydro-resveratrol ameliorates lung injury in rats with cerulein-induced acute pancreatitis. Phytother. Res. 30, 663-670. doi: 10.1002/ptr.5576

Liu, G., Zhang, J., Chen, H., Wang, C., Qiu, Y., Liu, Y., et al. (2014). Effects and mechanisms of alveolar type II epithelial cell apoptosis in severe pancreatitisinduced acute lung injury. Exp. Ther. Med. 7, 565-572. doi: 10.3892/etm.2013. 1453

Liu, J., Mu, J., Zheng, C., Chen, X., Guo, Z., Huang, C., et al. (2016). Systemspharmacology dissection of traditional chinese medicine compound saffron formula reveals multi-scale treatment strategy for cardiovascular diseases. Sci. Rep. 6:19809. doi: 10.1038/srep19809
Liu, X. B., Jiang, J. M., Huang, Z. W., Tian, B. L., Hu, W. M., Xia, Q., et al. (2004). Clinical study on the treatment of severe acute pancreatitis by integrated traditional Chinese medicine and Western medicine. Sichuan Da Xue Xue Bao Yi Xue Ban 35, 204-208.

Lowenfels, A. B., Lankisch, P. G., and Maisonneuve, P. (2000). What is the risk of biliary pancreatitis in patients with gallstones? Gastroenterology 119, 879-880. doi: 10.1053/gast.2000.17934

Lur, G., Haynes, L. P., Prior, I. A., Gerasimenko, O. V., Feske, S., Petersen, O. H., et al. (2009). Ribosome-free terminals of rough ER allow formation of STIM1 puncta and segregation of STIM1 from IP(3) receptors. Curr. Biol. 19, 1648-1653. doi: 10.1016/j.cub.2009.07.072

Ma, Q., Zhang, M., Wang, Z., Ma, Z., and Sha, H. (2011). The beneficial effect of resveratrol on severe acute pancreatitis. Ann. N. Y. Acad. Sci. 1215, 96-102. doi: 10.1111/j.1749-6632.2010.05847.x

Ma, Z. H., Ma, Q. Y., Wang, L. C., Sha, H. C., Wu, S. L., and Zhang, M. (2005). Effect of resveratrol on peritoneal macrophages in rats with severe acute pancreatitis. Inflamm. Res. 54, 522-527. doi: 10.1007/s00011-005-1388-z

Maleth, J., and Hegyi, P. (2014). Calcium signaling in pancreatic ductal epithelial cells: an old friend and a nasty enemy. Cell Calcium 55, 337-345. doi: 10.1016/j. ceca.2014.02.004

Meng, Y., Ma, Q. Y., Kou, X. P., and Xu, J. (2005a). Effect of resveratrol on activation of nuclear factor kappa-B and inflammatory factors in rat model of acute pancreatitis. World J. Gastroenterol. 11, 525-528.

Meng, Y., Zhang, M., Xu, J., Liu, X. M., and Ma, Q. Y. (2005b). Effect of resveratrol on microcirculation disorder and lung injury following severe acute pancreatitis in rats. World J. Gastroenterol. 11, 433-435.

Morishima, N., Nakanishi, K., Takenouchi, H., Shibata, T., and Yasuhiko, Y. (2002). An endoplasmic reticulum stress-specific caspase cascade in apoptosis. Cytochrome c-independent activation of caspase-9 by caspase-12. J. Biol. Chem. 277, 34287-34294. doi: 10.1074/jbc.M204973200

Mukherjee, R., Mareninova, O. A., Odinokova, I. V., Huang, W., Murphy, J., Chvanov, M., et al. (2016). Mechanism of mitochondrial permeability transition pore induction and damage in the pancreas: inhibition prevents acute pancreatitis by protecting production of ATP. Gut 65, 1333-1346. doi: 10.1136/ gutjnl-2014-308553

Nakagawa, T., and Yuan, J. (2000). Cross-talk between two cysteine protease families. Activation of caspase-12 by calpain in apoptosis. J. Cell Biol. 150, 887-894. doi: $10.1083 /$ jcb.150.4.887

Nauseef, W. M., and Borregaard, N. (2014). Neutrophils at work. Nat. Immunol. 15, 602-611. doi: 10.1038/ni.2921

Ni, Q., Sun, K., Chen, G., and Shang, D. (2014a). In vitro effects of emodin on peritoneal macrophages that express membrane-bound CD14 protein in a rat model of severe acute pancreatitis/systemic inflammatory response syndrome. Mol. Med. Rep. 9, 355-359. doi: 10.3892/mmr.2013.1771

Ni, Q., Zhang, W., Sun, K., Yin, C., An, J., and Shang, D. (2014b). In vitro effects of emodin on peritoneal macrophage intercellular adhesion molecule-3 in a rat model of severe acute pancreatitis/systemic inflammatory response syndrome. Biomed. Rep. 2, 63-68. doi: 10.3892/br.2013.178

Ning, J. W., Ji, F., Luo, D. D., Yang, C. Y., and Wang, L. J. (2009). Effects of emodin on cell apoptosis of intestinal mucosa and serum leptin in rats with severe acute pancreatitis. Zhong Xi Yi Jie He Xue Bao 7, 1167-1173. doi: 10. 3736/jcim 20091215

Normile, D. (2003). Asian medicine. The new face of traditional Chinese medicine. Science 299, 188-190. doi: 10.1126/science.299.5604.188

Park, B. K., Chung, J. B., Lee, J. H., Suh, J. H., Park, S. W., Song, S. Y., et al. (2003). Role of oxygen free radicals in patients with acute pancreatitis. World J. Gastroenterol. 9, 2266-2269. doi: 10.3748/wjg.v9.i10.2266

Peng, X. H., Huang, W., Zhu, L., Xia, Q., and Tang, W. F. (2013). Effects of micron Chinese herbal Liu-He-Dan ointment on inflammation and oxidative stress in rats with acute pancreatitis. Sichuan Da Xue Xue Bao Yi Xue Ban 44, 978-981.

Perez, S., Pereda, J., Sabater, L., and Sastre, J. (2015). Redox signaling in acute pancreatitis. Redox Biol. 5, 1-14. doi: 10.1016/j.redox.2015.01.014

Qin, D. P., Wei, X., Fang, G. D., Yang, F., and Lai, D. P. (2015). Intervention effect of modified Dachengqi decoction on intestinal mucosal barrier of severe acute pancreatitis model rats. Zhongguo Zhong Xi Yi Jie He Za Zhi 35, 1482-1489.

Qiong, W., Yiping, W., Jinlin, Y., Tao, G., Zhen, G., and Pengcheng, Z. (2005). Chinese medicinal herbs for acute pancreatitis. Cochrane Database Syst. Rev. CD003631. doi: 10.1002/14651858.CD003631.pub2 
Ren, Y. Y., Gong, H. L., Tang, W. F., Wan, M. H., Zhao, J. L., and Huang, X. (2009). Dachengqi decoction induces pancreatic acinar cell apoptosis in experimental acute pancreatitis in rats. Zhong Xi Yi Jie He Xue Bao 7, 651-656. doi: 10.3736/ jcim20090709

Riesle, E., Friess, H., Zhao, L., Wagner, M., Uhl, W., Baczako, K., et al. (1997). Increased expression of transforming growth factor beta s after acute oedematous pancreatitis in rats suggests a role in pancreatic repair. Gut 40, 73-79. doi: 10.1136/gut.40.1.73

Rotstein, O. D. (2014). Circulating cytokines in predicting development of severe acute pancreatitis. Crit. Care 18:575. doi: 10.1186/s13054-014-0575-0

Saluja, A. K., Donovan, E. A., Yamanaka, K., Yamaguchi, Y., Hofbauer, B., and Steer, M. L. (1997). Cerulein-induced in vitro activation of trypsinogen in rat pancreatic acini is mediated by cathepsin B. Gastroenterology 113, 304-310. doi: 10.1016/S0016-5085(97)70108-2

Scaffidi, P., Misteli, T., and Bianchi, M. E. (2002). Release of chromatin protein HMGB1 by necrotic cells triggers inflammation. Nature 418, 191-195. doi: 10.1038/nature00858

Schmitt, M., Klonowski-Stumpe, H., Eckert, M., Luthen, R., and Haussinger, D. (2004). Disruption of paracellular sealing is an early event in acute caeruleinpancreatitis. Pancreas 28, 181-190. doi: 10.1097/00006676-20040300000010

Schraml, B. U., Hildner, K., Ise, W., Lee, W. L., Smith, W. A., Solomon, B., et al. (2009). The AP-1 transcription factor Batf controls $\mathrm{T}(\mathrm{H}) 17$ differentiation. Nature 460, 405-409. doi: 10.1038/nature08114

Sendler, M., Dummer, A., Weiss, F. U., Kruger, B., Wartmann, T., ScharffetterKochanek, K., et al. (2013). Tumour necrosis factor alpha secretion induces protease activation and acinar cell necrosis in acute experimental pancreatitis in mice. Gut 62, 430-439. doi: 10.1136/gutjnl-2011-300771

Sha, H., Ma, Q., Jha, R. K., Wu, Z., Qingyuan, Z., Wang, Z., et al. (2013). Resveratrol suppresses microcirculatory disturbance in a rat model of severe acute pancreatitis. Cell Biochem. Biophys. 67, 1059-1065. doi: 10.1007/s12013013-9604-x

Sha, H., Ma, Q., Jha, R. K., Xu, F., Wang, L., Wang, Z., et al. (2008). Resveratrol ameliorates hepatic injury via the mitochondrial pathway in rats with severe acute pancreatitis. Eur. J. Pharmacol. 601, 136-142. doi: 10.1016/j.ejphar.2008. 10.017

Shehzad, A., Rehman, G., and Lee, Y. S. (2013). Curcumin in inflammatory diseases. Biofactors 39, 69-77. doi: 10.1002/biof.1066

Shen, X., and Li, W. Q. (2015). High-mobility group box 1 protein and its role in severe acute pancreatitis. World J. Gastroenterol. 21, 1424-1435. doi: 10.3748/ wjg.v21.i5.1424

Shen, Y. C., Chiou, W. F., Chou, Y. C., and Chen, C. F. (2003). Mechanisms in mediating the anti- inflammatory effects of baicalin and baicalein in human leukocytes. Eur. J. Pharmacol. 465, 171-181. doi: 10.1016/S0014-2999(03) 01378-5

Snyder, J. (2000). Pediatric gastrointestinal disease: pathophysiology, diagnosis and management. Gastroenterology 119:273. doi: 10.1016/S0016-5085(00) 70066-7

Szabolcs, A., Varga, I. S., Varga, C., Berko, A., Kaszaki, J., Letoha, T., et al. (2006). Beneficial effect of resveratrol on cholecystokinin-induced experimental pancreatitis. Eur. J. Pharmacol. 532, 187-193. doi: 10.1016/j.ejphar.2006.01.055

Takeyama, Y. (2005). Significance of apoptotic cell death in systemic complications with severe acute pancreatitis. J. Gastroenterol. 40, 1-10. doi: 10.1007/s00535004-1505-8

Tenner, S., Baillie, J., DeWitt, J., and Vege, S. S. (2013). American College of Gastroenterology guideline: management of acute pancreatitis. Am. J. Gastroenterol. 108, 1400-1415; 1416. doi: 10.1038/ajg.2013.218

Thomas, D. A., and Rosenthal, G. A. (1987). Toxicity and pharmacokinetics of the nonprotein amino acid L-canavanine in the rat. Toxicol. Appl. Pharmacol. 91, 395-405. doi: 10.1016/0041-008X(87)90061-5

Tian, H., Zhang, X., Wu, C., Chen, L., Ying, R., Ye, J., et al. (2009). Effects of Baicalin and Octreotide on the serum TNF-alpha level and apoptosis in multiple organs of rats with severe acute pancreatitis. Inflammation 32, 191-201. doi: 10.1007/s10753-009-9120-8

Tomkotter, L., Erbes, J., Trepte, C., Hinsch, A., Dupree, A., Bockhorn, M., et al. (2016). The effects of pancreatic microcirculatory disturbances on histopathologic tissue damage and the outcome in severe acute pancreatitis. Pancreas 45, 248-253. doi: 10.1097/mpa.0000000000000440
Tsang, S. W., Guan, Y. F., Wang, J., Bian, Z. X., and Zhang, H. J. (2016). Inhibition of pancreatic oxidative damage by stilbene derivative dihydro-resveratrol: implication for treatment of acute pancreatitis. Sci. Rep. 6:22859. doi: 10.1038/ srep22859

Tsukahara, Y., Morisaki, T., Horita, Y., Torisu, M., and Tanaka, M. (1999). Phospholipase A2 mediates nitric oxide production by alveolar macrophages and acute lung injury in pancreatitis. Ann. Surg. 229, 385-392. doi: 10.1097/ 00000658-199903000-00012

Ueda, S., Nakamura, H., Masutani, H., Sasada, T., Takabayashi, A., Yamaoka, Y., et al. (2002). Baicalin induces apoptosis via mitochondrial pathway as prooxidant. Mol. Immunol. 38, 781-791. doi: 10.1016/S0161-5890(01) 00115-8

Van Acker, G. J., Saluja, A. K., Bhagat, L., Singh, V. P., Song, A. M., and Steer, M. L. (2002). Cathepsin B inhibition prevents trypsinogen activation and reduces pancreatitis severity. Am. J. Physiol. Gastrointest. Liver Physiol. 283, G794-G800. doi: 10.1152/ajpgi.00363.2001

Wallig, M. A., Gould, D. H., and Fettman, M. J. (1988). Selective pancreato-toxicity in the rat induced by the naturally occurring plant nitrile 1-cyano-2-hydroxy3-butene. Food Chem. Toxicol. 26, 137-147. doi: 10.1016/0278-6915(88) 90110-X

Wang, G., Sun, B., Gao, Y., Meng, Q. H., and Jiang, H. C. (2007). The effect of emodin-assisted early enteral nutrition on severe acute pancreatitis and secondary hepatic injury. Mediators Inflamm. 2007:29638. doi: 10.1155/2007/ 29638

Wang, H., Zheng, H., Zhao, Z., and Chen, P. (2009). Effect of Chaihu Shihuang soup on blood serum level TNF, IL-6 and IL-10 of severe acute pancratitis (SAP). Zhongguo Zhong Yao Za Zhi 34, 1582-1584.

Wang, J., Chen, G., Gong, H., Huang, W., Long, D., and Tang, W. (2012). Amelioration of experimental acute pancreatitis with Dachengqi decoction via regulation of necrosis-apoptosis switch in the pancreatic acinar cell. PLOS ONE 7:e40160. doi: 10.1371/journal.pone.0040160

Wang, J., Li, C., Jiang, Y., Zheng, H., Li, D., Liang, Y., et al. (2016). Effect of ceramide-1-phosphate transfer protein on intestinal bacterial translocation in severe acute pancreatitis. Clin. Res. Hepatol. Gastroenterol. 41, 86-92. doi: 10. 1016/j.clinre.2016.08.003

Wang, L., Li, Y., Ma, Q., Liu, Y., Rui, Y. Y., Xue, P., et al. (2011). Chaiqin Chengqi decoction decreases IL-6 levels in patients with acute pancreatitis. J. Zhejiang Univ. Sci. B 12, 1034-1040. doi: 10.1631/jzus.B1000406

Wang, L., Ma, Q., Chen, X., Sha, H., and Ma, Z. (2008). Effects of resveratrol on calcium regulation in rats with severe acute pancreatitis. Eur. J. Pharmacol. 580, 271-276. doi: 10.1016/j.ejphar.2007.10.068

Wang, Z. C., Xue, P., and Huang, Z. W. (2009). Effect of an early application of chaiqin chengqi decoction in treating severe acute pancreatitis complicated with acute respiratory distress syndrome. Zhongguo Zhong Xi Yi Jie He Za Zhi 29, 322-324.

Wargo, K. A., Allman, E., and Ibrahim, F. (2010). A possible case of saw palmetto-induced pancreatitis. South. Med. J. 103, 683-685. doi: 10.1097/SMJ. 0b013e3181ele3ee

Wartmann, T., Mayerle, J., Kahne, T., Sahin-Toth, M., Ruthenburger, M., Matthias, R., et al. (2010). Cathepsin L inactivates human trypsinogen, whereas cathepsin L-deletion reduces the severity of pancreatitis in mice. Gastroenterology 138, 726-737. doi: 10.1053/j.gastro.2009.10.048

Weng, T. I., Wu, H. Y., Chen, B. L., and Liu, S. H. (2012). Honokiol attenuates the severity of acute pancreatitis and associated lung injury via acceleration of acinar cell apoptosis. Shock 37, 478-484. doi: 10.1097/SHK.0b013e3182 4653be

Whitcomb, D. C. (2006). Clinical practice. Acute pancreatitis. N. Engl. J. Med. 354, 2142-2150. doi: 10.1056/NEJMcp054958

Working Group IAP/APA Acute Pancreatitis Guidelines (2013). IAP/APA evidence-based guidelines for the management of acute pancreatitis. Pancreatology 13(4 Suppl. 2), e1-e15. doi: 10.1016/j.pan.2013.07.063

Wu, C. T., Li, Z. L., and Xiong, D. X. (1998). Relationship between enteric microecologic dysbiosis and bacterial translocation in acute necrotizing pancreatitis. World J. Gastroenterol. 4, 242-245. doi: 10.3748/wjg.v4. i3.242

Wu, J. S., Li, W. M., Chen, Y. N., Zhao, Q., and Chen, Q. F. (2016). Endoplasmic reticulum stress is activated in acute pancreatitis. J. Dig. Dis. 17, 295-303. doi: $10.1111 / 1751-2980.12347$ 
Wu, T. Y., Chang, F. R., Liou, J. R., Lo, I. W., Chung, T. C., Lee, L. Y., et al. (2016). Rapid HPLC quantification approach for detection of active constituents in modern combinatorial formula, San-Huang-Xie-Xin-Tang (SHXXT). Front. Pharmacol. 7:374. doi: 10.3389/fphar.2016.00374

Wu, W. U., Luo, R., Lin, Z., Xia, Q., and Xue, P. (2016). Key molecular mechanisms of Chaiqinchengqi decoction in alleviating the pulmonary albumin leakage caused by endotoxemia in severe acute pancreatitis rats. Evid. Based Complement. Alternat. Med. 2016:3265368. doi: 10.1155/2016/ 3265368

Wu, J. X., Xu, J. Y., and Yuan, Y. Z. (2000). Effect of emodin and sandostatin on metabolism of eicosanoids in acute necrotizing pancreatitis. World $J$. Gastroenterol. 6, 293-294.

Wu, L., Cai, B., Liu, X., and Cai, H. (2014). Emodin attenuates calcium overload and endoplasmic reticulum stress in AR42J rat pancreatic acinar cells. Mol. Med. Rep. 9, 267-272. doi: 10.3892/mmr.2013.1773

Wu, L., Cai, B., Zheng, S., Liu, X., Cai, H., and Li, H. (2013). Effect of emodin on endoplasmic reticulum stress in rats with severe acute pancreatitis. Inflammation 36, 1020-1029. doi: 10.1007/s10753-013-9634-y

Wu, W., Guo, J., Yang, X. N., Lin, Z. Q., Huang, Z., Xia, Q., et al. (2012). Effect of Chaiqinchengqi decoction on serum amyloid $\mathrm{A}$ in severe acute pancreatitis patients. Asian Pac. J. Trop. Med. 5, 901-905. doi: 10.1016/s1995-7645(12) 60168-0

Wu, Y., and Tang, L. (2016). Bcl-2 family proteins regulate apoptosis and epithelial to mesenchymal transition by calcium signals. Curr. Pharm. Des. 22, 4700-4704. doi: 10.2174/1381612822666160506125937

Xia, T., Jiang, C., Li, L., Wu, C., Chen, Q., and Liu, S. S. (2002). A study on permeability transition pore opening and cytochrome $\mathrm{c}$ release from mitochondria, induced by caspase-3 in vitro. FEBS Lett. 510, 62-66. doi: 10. 1016/S0014-5793(01)03228-8

Xia, X. M., Li, B. K., Xing, S. M., and Ruan, H. L. (2012). Emodin promoted pancreatic claudin-5 and occludin expression in experimental acute pancreatitis rats. World J. Gastroenterol. 18, 2132-2139. doi: 10.3748/wjg.v18.i17. 2132

Xia, X. M., Wang, F. Y., Wang, Z. K., Wan, H. J., Xu, W. A., and Lu, H. (2010). Emodin enhances alveolar epithelial barrier function in rats with experimental acute pancreatitis. World J. Gastroenterol. 16, 2994-3001. doi: 10.3748/wjg.v16. i24.2994

Xiang, H., Wang, G., Qu, J., Xia, S., Tao, X., Qi, B., et al. (2016). Yin-Chen-Hao Tang attenuates severe acute pancreatitis in rat: an experimental verification of in silico network target prediction. Front. Pharmacol. 7:378. doi: 10.3389/fphar. 2016.00378

Xiping, Z., Guanghua, F., Jinxian, H., Weihong, W., Rujun, X., Wei, Z., et al. (2010). Baicalin protects thymus of rats with severe acute pancreatitis. Inflammation 33, 157-165. doi: 10.1007/s10753-009-9169-4

Xiping, Z., Hua, T., Hanqing, C., Li, C., Binyan, Y., and Jing, M. (2009a). Effects of Baicalin on inflammatory mediators and pancreatic acinar cell apoptosis in rats with sever acute pancreatitis. J. Res. Med. Sci. 14, 19-27.

Xiping, Z., Hua, T., Hanqing, C., Li, C., Zhiwei, W., Keyi, W., et al. (2007). The protecting effects and mechanisms of Baicalin and Octreotide on heart injury in rats with SAP. Mediators Inflamm. 2007:19469. doi: 10.1155/2007/ 19469

Xiping, Z., Hua, T., Jie, Z., Li, C., Yang, C., Bei, L., et al. (2009b). Influence of baicalin on TNF-alpha mRNA, caspase-3 and P-selectin expression in pancreatic tissue of rats with severe acute pancreatitis. Indian J. Gastroenterol. 28, 131-135. doi: 10.1007/s12664-009-0047-7

Xu, J., Huang, B., Wang, Y., Tong, C., Xie, P., Fan, R., et al. (2016). Emodin ameliorates acute lung injury induced by severe acute pancreatitis through the up-regulated expressions of AQP1 and AQP5 in lung. Clin. Exp. Pharmacol. Physiol. 43, 1071-1079. doi: 10.1111/1440-1681.12627

Xue, D., Zhang, W., Zhang, Y., Wang, H., Zheng, B., and Shi, X. (2006). Adjusting effects of baicalin for nuclear factor-kappaB and tumor necrosis factor-alpha on rats with caerulein-induced acute pancreatitis. Mediators Inflamm. 2006:26295. doi: $10.1155 / \mathrm{mi} / 2006 / 26295$

Xue, P., Deng, L. H., Zhang, Z. D., Yang, X. N., Xia, Q., Xiang, D. K., et al. (2008). Effect of Chaiqinchengqi decoction on sarco/endoplasmic reticulum $\mathrm{Ca}^{2+}$-ATPase mRNA expression of pancreatic tissues in acute pancreatitis rats. World J. Gastroenterol. 14, 2343-2348. doi: 10.3748/wjg.14. 2343
Xue, P., Guo, J., Yang, X. N., Huang, W., and Xia, Q. (2014). Changes of neuronal acetylcholine receptor alpha 7 of peritoneal macrophage in experimental acute pancreatitis treated by Chaiqin Chengqi Decoction (). Chin. J. Integr. Med. 20, 770-775. doi: 10.1007/s11655-013-1661-x

Yadav, D., Eigenbrodt, M. L., Briggs, M. J., Williams, D. K., and Wiseman, E. J. (2007). Pancreatitis: prevalence and risk factors among male veterans in a detoxification program. Pancreas 34, 390-398. doi: 10.1097/mpa. 0b013e318040b332

Yadav, D., and Lowenfels, A. B. (2006). Trends in the epidemiology of the first attack of acute pancreatitis: a systematic review. Pancreas 33, 323-330. doi: 10.1097/01.mpa.0000236733.31617.52

Yadav, D., and Lowenfels, A. B. (2013). The epidemiology of pancreatitis and pancreatic cancer. Gastroenterology 144, 1252-1261. doi: 10.1053/j.gastro.2013. 01.068

Yadav, D., O'Connell, M., and Papachristou, G. I. (2012). Natural history following the first attack of acute pancreatitis. Am. J. Gastroenterol. 107, 1096-1103. doi: 10.1038/ajg.2012.126

Yang, D. Y., Duan, S. B., and Aili, J. T. (2009). Effect of qingyi decoction in treating severe acute pancreatitis and its impacts on blood level of tumor necrosis factoralpha, interleukin-6 and inteleukin-8. Zhongguo Zhong Xi Yi Jie He Za Zhi 29, 1122-1124.

Yang, H. L., Lin, M. W., Korivi, M., Wu, J. J., Liao, C. H., Chang, C. T., et al. (2016). Coenzyme Q0 regulates NFkappaB/AP-1 activation and enhances Nrf2 stabilization in attenuation of LPS-induced inflammation and redox imbalance: evidence from in vitro and in vivo studies. Biochim. Biophys. Acta 1859, 246-261. doi: 10.1016/j.bbagrm.2015.11.001

Yang, Y. S., Chen, K., Xie, W. R., and Wang, H. (2015). Effect of Qingyi Granule on HMGB1 expression in liver and renal tissues of severe acute pancreatitis rats. Zhongguo Zhong Xi Yi Jie He Za Zhi 35, 1367-1372.

Yang, Y. S., Chen, K., Ye, S., Shi, X. L., Du, Z. W., Cui, S. L., et al. (2013). Differentially expressed proteins of severe acute pancreatitis intervened by Qingyi granule. Zhongguo Zhong Xi Yi Jie He Za Zhi 33, 60-64.

Yang, Z. W., Meng, X. X., and Xu, P. (2015). Central role of neutrophil in the pathogenesis of severe acute pancreatitis. J. Cell Mol. Med. 19, 2513-2520. doi: $10.1111 / \mathrm{jcmm} .12639$

Yao, H., Liu, J., Xu, S., Zhu, Z., and Xu, J. (2016a). The structural modification of natural products for novel drug discovery. Expert Opin. Drug Discov. 12, 121-140. doi: 10.1080/17460441.2016.1272757

Yao, H., Qiao, Y. J., Zhao, Y. L., Tao, X. F., Xu, L. N., Yin, L. H., et al. (2016b). Herbal medicines and nonalcoholic fatty liver disease. World J. Gastroenterol. 22, 6890-6905. doi: 10.3748/wjg.v22.i30.6890

Yao, W. Y., Zhou, Y. F., Qian, A. H., Zhang, Y. P., Qiao, M. M., Zhai, Z. K., et al. (2015). Emodin has a protective effect in cases of severe acute pancreatitis via inhibition of nuclear factorkappaB activation resulting in antioxidation. Mol. Med. Rep. 11, 1416-1420. doi: 10.3892/mmr.2014.2789

Yin, J. T., Wan, B., Liu, D. D., Wan, S. X., Fu, H. Y., Wan, Y., et al. (2016). Emodin alleviates lung injury in rats with sepsis. J. Surg. Res. 202, 308-314. doi: 10.1016/j.jss.2015.12.049

Yokoe, M., Takada, T., Mayumi, T., Yoshida, M., Isaji, S., Wada, K., et al. (2015). Japanese guidelines for the management of acute pancreatitis: Japanese Guidelines 2015. J. Hepatobiliary Pancreat. Sci. 22, 405-432. doi: 10.1002/ jhbp. 259

Yu, J. H., and Kim, H. (2014). Oxidative stress and inflammatory signaling in cerulein pancreatitis. World J. Gastroenterol. 20, 17324-17329. doi: 10.3748/wjg. v20.i46.17324

Yu, W. G., Xu, G., Ren, G. J., Xu, X., Yuan, H. Q., Qi, X. L., et al. (2011). Preventive action of curcumin in experimental acute pancreatitis in mouse. Indian J. Med. Res. 134, 717-724. doi: 10.4103/0971-5916.91009

Zeng, Y., Wang, X., Zhang, W., Wu, K., and Ma, J. (2012). Hypertriglyceridemia aggravates ER stress and pathogenesis of acute pancreatitis. Hepatogastroenterology 59, 2318-2326. doi: 10.5754/hge12042

Zhang, J. W., Zhang, G. X., Chen, H. L., Liu, G. L., Owusu, L., Wang, Y. X., et al. (2015). Therapeutic effect of Qingyi decoction in severe acute pancreatitisinduced intestinal barrier injury. World J. Gastroenterol. 21, 3537-3546. doi: 10.3748/wjg.v21.i12.3537

Zhang, X., Feng, G., Weng, W., Liang, J., Lin, N., Cai, Y., et al. (2009a). Protective effects of baicalin and octreotide on intestinal mucosa of rats with severe acute pancreatitis. Turk. J. Gastroenterol. 20, 108-115. 
Zhang, X., Tian, H., Wu, C., Ye, Q., Jiang, X., Chen, L., et al. (2009b). Effect of baicalin on inflammatory mediator levels and microcirculation disturbance in rats with severe acute pancreatitis. Pancreas 38, 732-738. doi: 10.1097/MPA. 0b013e3181ad9735

Zhang, X. M., Ma, P. A., Sun, J. W., Duan, C. N., Hou, X. H., and Zhang, Y. C. (2014). Effect of Qingyi Chengqi Decoction on severe acute pancreatitis patients: a clinical study. Zhongguo Zhong Xi Yi Jie He Za Zhi 34, 31-34.

Zhang, X. P., Lin, Q., and Zhou, Y. F. (2007a). Progress of study on the relationship between mediators of inflammation and apoptosis in acute pancreatitis. Dig. Dis. Sci. 52, 1199-1205. doi: 10.1007/s10620-006-9388-6

Zhang, X. P., Tian, H., Lai, Y. H., Chen, L., Zhang, L., Cheng, Q. H., et al. (2007b). Protective effects and mechanisms of Baicalin and octreotide on renal injury of rats with severe acute pancreatitis. World J. Gastroenterol. 13, 5079-5089.

Zhang, X. P., Tian, H., Wu, D. J., Feng, G. H., Chen, L., Zhang, J., et al. (2009). Pathological changes in multiple organs of rats with severe acute pancreatitis treated by baicalin and octreotide. Hepatobiliary Pancreat. Dis. Int. 8, 85-92.

Zhang, X. P., Zhang, L., He, J. X., Zhang, R. P., Cheng, Q. H., Zhou, Y. F., et al. (2007c). Experimental study of therapeutic efficacy of Baicalin in rats with severe acute pancreatitis. World J. Gastroenterol. 13, 717-724.

Zhang, X. P., Zhang, J., Ren, Z., Feng, G. H., Zhu, W., Cai, Y., et al. (2008a). Study on protecting effects of baicalin and octreotide on hepatic injury in rats with severe acute pancreatitis. World J. Gastroenterol. 14, 6551-6559.

Zhang, X. P., Zhang, L., Yang, P., Zhang, R. P., and Cheng, Q. H. (2008b). Protective effects of baicalin and octreotide on multiple organ injury in severe acute pancreatitis. Dig. Dis. Sci. 53, 581-591. doi: 10.1007/s10620-007-9868-3

Zhang, Y., Zhu, L., Wang, J., Zhao, J., Zhao, X., Guo, H., et al. (2016). Formula compatibility identification of Dachengqi decoction based on the effects of absorbed components in cerulein-injured pancreatic AR42J cells. Evid. Based Complement. Alternat. Med. 2016:3198549. doi: 10.1155/2016/3198549

Zhong, K. (2015). Curcumin mediates a protective effect via TLR-4/NF-kappaB signaling pathway in rat model of severe acute pancreatitis. Cell Biochem. Biophys. 73, 175-180. doi: 10.1007/s12013-015-0664-y

Zhou, Z. G., and Chen, Y. D. (2002). Influencing factors of pancreatic microcirculatory impairment in acute pancreatitis. World J. Gastroenterol. 8, 406-412. doi: 10.3748/wjg.v8.i3.406

Zhou, Z. G., Chen, Y. Q., Liu, X. B., Hu, W. M., Tian, B. L., and Chen, H. Q. (2004). Changes of cytosolic $\left[\mathrm{Ca}^{2+}\right] \mathrm{i}$ in neutrophils in pancreatic microcirculation of rats with caerulein-induced acute pancreatitis under fluid shear stress. World J. Gastroenterol. 10, 3185-3187. doi: 10.3748/wjg.v10.i21.3185

Zhu, F. S., Zhu, G. Y., Huang, D. P., Shen, X. Y., Yang, C. Q., and Gao, H. J. (2014). Effect of Qingyi decoction on gene expression profiles of severe acute pancreatitis rats by gene chip technique. Zhongguo Zhong Xi Yi Jie $\mathrm{He} \mathrm{Za} Z \mathrm{Zhi}$ 34, 51-55.

Conflict of Interest Statement: The authors declare that the research was conducted in the absence of any commercial or financial relationships that could be construed as a potential conflict of interest.

Copyright (C) 2017 Xiang, Zhang, Qi, Tao, Xia, Song, Qu and Shang. This is an open-access article distributed under the terms of the Creative Commons Attribution License (CC BY). The use, distribution or reproduction in other forums is permitted, provided the original author(s) or licensor are credited and that the original publication in this journal is cited, in accordance with accepted academic practice. No use, distribution or reproduction is permitted which does not comply with these terms. 